\title{
SUBMILLIMETER INTERFEROMETRY OF THE LUMINOUS INFRARED GALAXY NGC 4418: A HIDDEN HOT NUCLEUS WITH AN INFLOW AND AN OUTFLOW
}

\author{
Kazushi Sakamoto ${ }^{1}$, Susanne Aalto ${ }^{2}$, Francesco Costagliola ${ }^{2}$, Sergio Martín ${ }^{3}$, \\ Youichi Ohyama ${ }^{1}$, Martina C. Wiedner ${ }^{4}$, And David J. Wilner ${ }^{5}$ \\ ${ }^{1}$ Academia Sinica, Institute of Astronomy and Astrophysics, Taiwan \\ ${ }^{2}$ Department of Earth and Space Sciences, Chalmers University of Technology, Onsala Space Observatory, Onsala, Sweden \\ ${ }^{3}$ European Southern Observatory, Santiago, Chile \\ ${ }^{4}$ LERMA and UMR 8112 du CNRS, Observatoire de Paris, 61 Av. de l'Observatoire, F-75014 Paris, France \\ ${ }^{5}$ Harvard-Smithsonian Center for Astrophysics, Cambridge, MA, USA \\ Received 2012 October 16; accepted 2012 December 6; published 2013 January 24
}

\begin{abstract}
We have observed the nucleus of the nearby luminous infrared galaxy NGC 4418 with subarcsec resolution at 860 and $450 \mu \mathrm{m}$ for the first time to characterize its hidden power source. A $\sim 20 \mathrm{pc}\left(0^{\prime \prime} 1\right)$ hot dusty core was found inside a $100 \mathrm{pc}$ scale concentration of molecular gas at the galactic center. The $860 \mu \mathrm{m}$ continuum core has a deconvolved (peak) brightness temperature of $120-210 \mathrm{~K}$. The $\mathrm{CO}(3-2)$ peak brightness temperature there is as high as $90 \mathrm{~K}$ at $50 \mathrm{pc}$ resolution. The core has a bolometric luminosity of about $10^{11} L_{\odot}$, which accounts for most of the galaxy luminosity. It is Compton thick $\left(N_{\mathrm{H}} \gtrsim 10^{25} \mathrm{~cm}^{-2}\right)$ and has a high luminosity-to-mass ratio $(L / M) \sim 500 L_{\odot} M_{\odot}{ }^{-1}$ as well as a high luminosity surface density $10^{8.5 \pm 0.5} L_{\odot} \mathrm{pc}^{-2}$. These parameters are consistent with an active galactic nucleus to be the main luminosity source (with an Eddington ratio about 0.3), while they can be also due to a young starburst near its maximum $L / M$. We also found an optical color (reddening) feature that we attribute to an outflow cone emanating from the nucleus. The hidden hot nucleus thus shows evidence of both an inflow, previously seen with absorption lines, and the new outflow reported here in a different direction. The nucleus must be rapidly evolving with these gas flows.
\end{abstract}

Key words: galaxies: active - galaxies: evolution - galaxies: individual (NGC 4418) - galaxies: ISM

Online-only material: color figures

\section{INTRODUCTION}

There are galaxies hiding a compact luminous source in their nuclei and NGC 4418 is their local prototype. The extremely extinguished power source in this early-type disk galaxy was first recognized by Roche et al. (1986), who singled out the galaxy for its infrared brightness. They found very deep silicate absorption at $9.7 \mu \mathrm{m}$ toward the infrared-bright nucleus and deduced that the source of the infrared luminosity $\left(\sim 10^{11} L_{\odot}\right)$ is completely obscured by a dust shroud of $A_{V} \gg 50 \mathrm{mag}$. They further inferred from the weakness of line features in the infrared and optical spectra that the luminosity source is an accreting massive black hole (i.e., active galactic nucleus or AGN), although they also mentioned a nascent starburst embedded within a dusty cocoon as a possible alternative.

Subsequent observations to be reviewed in Section 1.1 have supported the presence of a deeply buried compact luminosity source, although its nature is still debated. NGC 4418 is the most absorption-dominated galaxy in the mid-infrared diagnostic diagram of Spoon et al. (2007). Its location on the diagonal branch in the diagram suggests that the luminosity source is almost completely covered by opaque dust. Usual spectroscopic signs of AGN can be completely extinguished by this heavy absorption. Hence, the main piece of evidence for a hidden AGN, among other indirect ones, has been the compactness of the source inferred from the absorption depth. It has been argued that dust is unlikely to cover an extended starburst so completely as to cause the deep absorption features (Roche et al. 1986; Dudley \& Wynn-Williams 1997; Spoon et al. 2001). On the other hand, the arguments for very young starburst are based on the low radio to far-infrared ratio and the detection of a warm molecular gas absorber toward the nucleus. The former ratio is lower than in radio-quiet AGNs and is consistent with a starburst in the pre-supernova age $(<5 \mathrm{Myr}$; Roussel et al. 2003). The absorbing molecules appear to be in the region where $\mathrm{X}$-rays would have destroyed them if the heating is due to an AGN (Lahuis et al. 2007).

The true nature of the luminous nucleus is important because this type of heavily extinguished nuclei could double the number of local AGNs (Maiolino et al. 2003) or it could represent a young phase of unusually intense and compact starburst $(\ll 100$ pc). Luminous hidden nuclei of this kind are also important because they may be in a short transition phase to unobscured AGNs, i.e., in the process of removing the obscuring gas (e.g., through an AGN-driven outflow; Sanders et al. 1988; Fischer et al. 2010). Such feedback is regarded as a key process in galaxy evolution and hence requires detailed study (Hopkins et al. 2006; Weiner et al. 2009).

A new approach to characterize and diagnose the compact, luminous, and extinguished nuclei of this kind is through submillimeter-wave observations at high spatial resolution. Submillimeter observations directly probe the thermal dust emission that constitutes most of the bolometric luminosity of such a nucleus. Unlike optical and infrared observations, one can look deep into the obscuring material since dust opacity decreases at longer wavelengths. Radio interferometry is available in the submillimeter to achieve subarcsec resolution that is currently unavailable in far-IR where the radiation of such a nucleus peaks. It is possible to estimate the bolometric luminosity and luminosity density of the nucleus using the Stefan-Boltzmann law by measuring the source size and brightness temperature at a frequency where the nucleus has a photosphere. One can also probe the nucleus by measuring its mass from rotation of circumnuclear molecular gas, which can be observed using submillimeter 


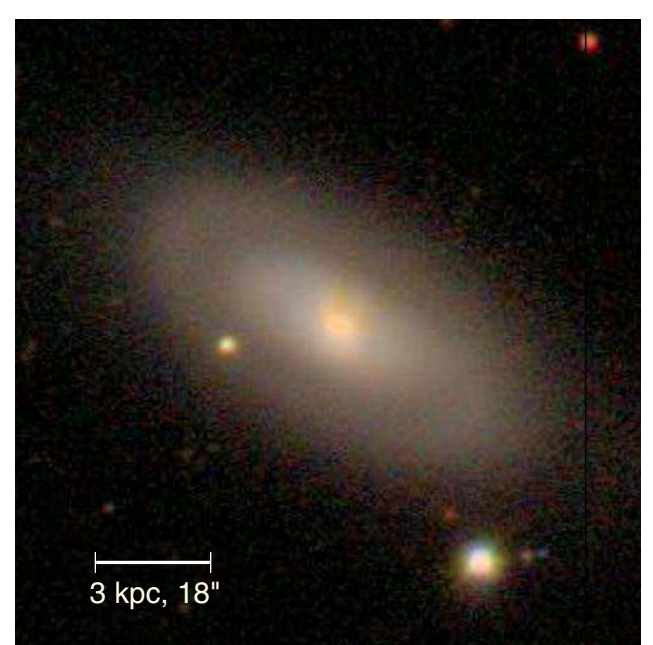

Figure 1. NGC 4418 optical image from the Sloan Digital Sky Survey $(0.3-1 \mu \mathrm{m}$ composite). The image size is $100^{\prime \prime}(16.5 \mathrm{kpc})$ on a side. North is up and east is to the left.

(A color version of this figure is available in the online journal.)

molecular lines. The luminosity-to-mass ratio, $L_{\mathrm{bol}} / M_{\mathrm{dyn}}$, so obtained will further constrain the nature of the nucleus since an AGN can have a higher $L / M$ than stars. Molecular lines can provide additional information on temperature, density, chemical composition of the interstellar medium (ISM), and on the radial motion of the gas affecting the evolution of the dusty shroud. We had applied this approach to the ultraluminous infrared galaxy Arp 220, which shares some of the above-mentioned characteristics of NGC 4418 including the deep silicate absorption. We found that the brighter one of the twin nuclei of Arp 220 has $L_{\text {bol }} \geqslant 2 \times 10^{11} L_{\odot}$ and $L_{\text {bol }} / M_{\text {dyn }} \gtrsim 400 L_{\odot} / M_{\odot}$ in the central 80 pc (Sakamoto et al. 2008, hereafter S08) and that each nucleus has a molecular outflow causing molecular P-Cygni profiles (Sakamoto et al. 2009).

In this paper, we apply the same approach to NGC 4418 to quantitatively constrain the nature of the deeply extinguished nucleus through submillimeter high-resolution observations. We made the first subarcsec resolution imaging of the galaxy in the submillimeter band, observing $860 \mu \mathrm{m}$ continuum and molecular lines including $\mathrm{CO}(3-2), \mathrm{HCN}(4-3)$, and $\mathrm{HCO}^{+}(4-3)$ and also imaging the nucleus with $450 \mu \mathrm{m}$ continuum. We also analyzed optical multi-color images to probe circumnuclear dust distribution for signs of disturbance and feedback. The obtained parameters of the nucleus are similar to the ones in Arp 220. They are consistent with any of the following being energetically dominant: an AGN, a young and compact starburst, and an AGNcompact starburst combination. Our results support the previous studies using an independent method and indicate a possible path to break the degeneracy about the luminosity source with future observations of higher quality. We also found a sign of outflow from the nucleus in the optical data. In the following, we briefly review properties of NGC 4418 and previous studies on the hidden nucleus in Section 1.1. We describe our Submillimeter Array (SMA) observations in Section 2 and present the results in Section 3, where we also report our examination of optical images of the galaxy. The results are discussed in Section 4 and the conclusions are summarized in Section 5. Our companion paper reports high-resolution $1 \mathrm{~mm}$ and $\mathrm{cm}$-wave observations of the same galaxy (Costagliola et al. 2012, hereafter C12).
Table 1

NGC 4418 Parameters

\begin{tabular}{lcc}
\hline \hline Parameter & Value & Note \\
\hline R.A. $(\mathrm{J} 2000)$ & $12^{\mathrm{h}} 26^{\mathrm{m}} 54^{\mathrm{s}} .612$ & $(1)$ \\
Decl. $(\mathrm{J} 2000)$ & $-00^{\circ} 52^{\prime} 39^{\prime \prime} .41$ & $(1)$ \\
$V_{\text {sys }}\left(\mathrm{km} \mathrm{s}^{-1}\right)$ & 2100 & $(2)$ \\
$D(\mathrm{Mpc})$ & 34 & $(3)$ \\
Scale. $1^{\prime \prime}$ in pc & 165 & \\
$L_{8-1000 \mu \mathrm{m}}\left(L_{\odot}\right)$ & $10^{11.1}$ & $(4)$ \\
$L_{\mathrm{K}}\left(L_{\odot \mathrm{K}}\right)$ & $10^{10.3}$ & $(5)$ \\
$L_{\mathrm{B}}\left(L_{\odot \mathrm{B}}\right)$ & $10^{9.8}$ & $(6)$ \\
Hubble type & $\left(\mathrm{R}^{\prime}\right) \mathrm{SAB}(\mathrm{s}) \mathrm{a}$ & $(6)$ \\
P.A. $\left({ }^{\circ}\right)$ & 60 & $(8)$ \\
$i\left(^{\circ}\right)$ & 62 & $(8)$ \\
\hline
\end{tabular}

Notes. (1) Position of the $860 \mu \mathrm{m}$ continuum nucleus measured in this work. The absolute positional uncertainty is estimated to be 0. '. 02 from the observations of a nearby quasar. Our pointing position was R.A. $=12^{\mathrm{h}} 26^{\mathrm{m}} 54^{\mathrm{s}} \cdot 620$, decl. $=$ $-00^{\circ} 52^{\prime} 39^{\prime \prime} .40$ taken from the Sloan Digital Sky Survey Data Release 6 through the NASA Extragalactic Database. (2) Centroid velocity of $\mathrm{CO}(2-1)$ spectrum (S10). Its formal uncertainty from the Gaussian fit is less than $1 \mathrm{~km} \mathrm{~s}^{-1}$, but see Section 3.4. (3) The galaxy distance we adopt. For $V_{\text {sys }}$ (radio, LSR) $=$ $2100 \mathrm{~km} \mathrm{~s}^{-1}$, the galaxy redshift corrected for local flow in the way of Mould et al. (2000) corresponds to an angular size and luminosity distance of 33.5 and $34.1 \mathrm{Mpc}$, respectively, for $H_{0}=73 \mathrm{~km} \mathrm{~s}^{-1} \mathrm{Mpc}^{-1}, \Omega_{\text {matter }}=0.73$, and $\Omega_{\text {vacuum }}=0.27$. (4) From the IRAS flux measurements in Sanders et al. (2003) and the formula in Table 1 of Sanders \& Mirabel (1996). (5) Two Micron All Sky Survey, as listed in the NASA Extragalactic Database. (6) de Vaucouleurs et al. (1991). (7) Position angle of the major axis from the Two Micron All Sky Survey Extended Source Catalogue (2MASS XSC; Jarrett et al. 2000). (8) Inclination of the galaxy disk. The minor to major axis ratio of $b / a=0.50$ in 2MASS XSC is translated to inclination assuming the intrinsic (edge-on) axial ratio of 0.2.

\subsection{NGC 4418}

Studies on NGC 4418 and its nucleus since Roche et al. (1986) are summarized below. (To guide readers, an optical image and key parameters of the galaxy are in Figure 1 and Table 1, respectively. We adopt the galaxy distance of $34 \mathrm{Mpc}$ at which $1^{\prime \prime}$ is 165 pc.) (1) A high infrared-to-optical luminosity ratio $L_{\mathrm{IR}} / L_{\mathrm{B}} \approx 20$ (Armus et al. 1987; Young et al. 1989) and the shape of the spectral energy distribution (SED) suggest that most of the luminosity of the galaxy is absorbed and reradiated by dust as thermal emission (Roche \& Chandler 1993). (2) NGC 4418 has warm mid-IR and far-IR colors $S(25 \mu \mathrm{m}) / S(60 \mu \mathrm{m})=$ 0.220 and $S(60 \mu \mathrm{m}) / S(100 \mu \mathrm{m})=1.37$ (Sanders et al. 2003). The former puts the galaxy in the "warm" population that often host active nuclei (de Grijp et al. 1985; Sanders et al. 1988). The latter also suggests warm dust (color temperature of $\sim 50 \mathrm{~K}$ ) indicative of reprocessed dust emission. (3) The deep absorption has been confirmed by further spectroscopy and modeling; the estimated opacity is $\tau_{9.7 \mu \mathrm{m}} \approx 7$ or $A_{V} \sim 100 \mathrm{mag}$ (Roche et al. 1986; Dudley \& Wynn-Williams 1997; Spoon et al. 2001; Siebenmorgen et al. 2008). A radial gradient of temperature in the nucleus may also contribute to the deep $10 \mu \mathrm{m}$ depression in the same way as Kwan \& Scoville (1976) modeled for protostars (Dudley \& Wynn-Williams 1997). It is also suggested from a shallow SED slope in the FIR that the dust has a high enough column density to be optically thick even at or beyond $100 \mu \mathrm{m}$ (Roche \& Chandler 1993; Lisenfeld et al. 2000). Likely related to this is an unusually low ratio of the [C II] $158 \mu \mathrm{m}$ line to far-IR luminosity in NGC 4418 (Malhotra et al. 1997; GraciáCarpio et al. 2011). (4) The compactness of the infrared nucleus has been directly confirmed with mid-IR imaging at subarcsec resolutions (Wynn-Williams \& Becklin 1993; Evans et al. 2003; 
Table 2

Log of SMA Observations

\begin{tabular}{|c|c|c|c|c|c|c|c|c|c|}
\hline \multirow{2}{*}{$\begin{array}{l}\text { No. } \\
\text { (1) }\end{array}$} & \multirow{2}{*}{$\begin{array}{l}\text { UT Date } \\
\text { (2) }\end{array}$} & \multirow{2}{*}{$\begin{array}{c}f_{\mathrm{LO}} \\
(\mathrm{GHz}) \\
(3)\end{array}$} & \multirow{2}{*}{$\begin{array}{l}N_{\text {ant }} \\
\text { (4) }\end{array}$} & \multicolumn{2}{|c|}{ Array Configuration } & \multirow{2}{*}{$\begin{array}{c}L_{\text {baseline }} \\
\text { (m) } \\
(7)\end{array}$} & \multirow{2}{*}{$\begin{array}{c}\tau_{225} \\
(8)\end{array}$} & \multirow{2}{*}{$\begin{array}{c}\left\langle T_{\text {sys }}\right\rangle \\
(\mathrm{K}) \\
(9)\end{array}$} & \multirow{2}{*}{$\begin{array}{l}T_{\text {obs }} \\
(\mathrm{hr}) \\
(10)\end{array}$} \\
\hline & & & & $\begin{array}{c}\text { Name } \\
(5)\end{array}$ & $\begin{array}{c}\text { Pads } \\
(6)\end{array}$ & & & & \\
\hline 1 & 2009 Mar 3 & 348.742 & 7 & VEX & $1,12,16,17,19,20,21$ & $40-509$ & 0.07 & 237 & 4.6 \\
\hline 2 & 2010 Mar 3 & 347.698 & 6 & VEX & $1,16,17,18,19,20$ & $85-509$ & 0.04 & 153 & 4.3 \\
\hline $2^{\prime}$ & 2010 Mar 3 & 663.041 & 5 & VEX & $1,17,18,19,20$ & $110-458$ & 0.04 & 1228 & 3.8 \\
\hline 3 & 2010 Mar 28 & 347.698 & 6 & $\mathrm{SC}$ & $1,2,3,4,5,6$ & $6-25$ & 0.05 & 204 & 2.1 \\
\hline
\end{tabular}

Notes. Column 3: frequency of the local oscillator. Upper (lower) sideband is from 4.0 to $6.0 \mathrm{GHz}$ above (below) this frequency for the first two tracks and from 4.0 to $8.0 \mathrm{GHz}$ for the third and fourth tracks. Column 4: number of available antennas. Column 5: SMA antenna configuration. VEX = very extended, EXT = extended, SC = subcompact. Column 6: antenna locations. See Ho et al. (2004) for a map with the numeric keys. Column 7: range of projected length of baselines for NGC 4418. Column 8: zenith opacity at $225 \mathrm{GHz}$ measured at the Caltech Submillimeter Observatory adjacent to the SMA. Column 9: median double sideband (DSB) system temperature toward NGC 4418. Column 10: total integration time on the galaxy.

Siebenmorgen et al. 2008). According to the Keck imaging by Evans et al. (2003), the $10 \mu \mathrm{m}$ size of the nucleus is $00^{\prime \prime} 3$ and the $25 \mu \mathrm{m}$ size is less than $0^{\prime \prime} 6$, which is the diffraction limit of the $10 \mathrm{~m}$ telescope. (5) The nucleus is also compact $\left(\sim 0^{\prime} .1-0^{\prime \prime} 4\right)$ and has a high brightness temperature $\left(\gtrsim 10^{5} \mathrm{~K}\right)$ at $\sim 2 \mathrm{GHz}$ (Condon et al. 1990; Kewley et al. 2000; Baan \& Klöckner 2006). Such a radio nucleus is either an active nucleus or a compact starburst or their hybrid (Smith et al. 1998). (6) Chandra X-ray observations "may imply the presence of a Compton-thick AGN," although the identification is "somewhat tentative" because of the limited photon statistics (Maiolino et al. 2003). (7) NGC 4418 has farIR excess compared to its radio strength, attributed either to a dust-enshrouded AGN or to a young, compact starburst (Kawara et al. 1990; Yun et al. 2001; Roussel et al. 2003). (8) Molecular gas concentration to the central $\leqslant 5^{\prime \prime}$ has been observed with interferometers (Imanishi et al. 2004; Dale et al. 2005; Sakamoto et al. 2010, hereafter S10; C12). The mass of the molecular gas will be about $1 \times 10^{9} M_{\odot}$ if the average conversion factor in our Galaxy (Hunter et al. 1997) applies to the observed CO(1-0) flux of $100 \pm 50 \mathrm{Jy} \mathrm{km} \mathrm{s}^{-1}$ (Kawara et al. 1990; Sanders et al. 1991; Young et al. 1995; Dale et al. 2005; Albrecht et al. 2007). NGC 4418 has a high infrared luminosity-to-molecular gas mass ratio of $L_{\mathrm{IR}} / M_{\text {mol }} \sim 100 L_{\odot} / M_{\odot}$ for the above $M_{\text {mol }}$ (Kawara et al. 1990; Sanders et al. 1991). High-excitation molecular lines with excitation temperatures above $100 \mathrm{~K}$ have been detected toward the nucleus (Aalto et al. 2007; Lahuis et al. 2007; Costagliola \& Aalto 2010; S10; González-Alfonso et al. 2012b; C12). The last four publications report vibrationally excited molecules and the last three report lines involving energy levels as high as $\sim 1000 \mathrm{~K}$. (9) Redshifted absorption lines have been found toward the nucleus by González-Alfonso et al. (2012b) in the mid-infrared $\mathrm{O}$ I and $\mathrm{OH}$ lines and by $\mathrm{C} 12$ in the $\mathrm{H}$ I $21 \mathrm{~cm}$ line. These are attributed to a gas inflow.

\section{SUBMILLIMETER ARRAY OBSERVATIONS}

\section{1. $350 \mathrm{GHz}(860 \mu \mathrm{m})$ Observations}

We observed NGC 4418 in 2009, 2010, and 2012 at around $350 \mathrm{GHz}(860 \mu \mathrm{m})$ using the SMA. ${ }^{6}$ We used the subcompact (SC), extended (EXT), and very extended (VEX) array configurations. Observational parameters are in Table 2. The data from

\footnotetext{
6 The SMA is a joint project between the Smithsonian Astrophysical Observatory and the Academia Sinica Institute of Astronomy and Astrophysics, and is funded by the Smithsonian Institution and the Academia Sinica.
}

the SC configuration were already reported in S10 and are combined here with the new data. The overall coverage of baseline lengths is $6-509 \mathrm{~m}$ or $7-590 \mathrm{k} \lambda$ at $350 \mathrm{GHz}$ for the galaxy. We simultaneously observed $\mathrm{CO}(3-2)$ and $\mathrm{HCO}^{+}(4-3)$ lines in 2009 and $\mathrm{CO}(3-2)$ and $\mathrm{HCN}(4-3)$ in 2010 using $2 \mathrm{GHz}$ of bandwidth. We used in the EXT and SC configurations $4 \mathrm{GHz}$ bandwidth to simultaneously observe $\mathrm{CO}(3-2), \mathrm{HCO}^{+}(4-3)$, and $\mathrm{HCN}(4-3)$. The SMA primary beam covers about $34^{\prime \prime}(5.6 \mathrm{kpc})$ in FWHM at our observing frequencies. The SMA spectrocorrelator was configured to the resolution of $3.25 \mathrm{MHz}$ (about $3 \mathrm{~km} \mathrm{~s}^{-1}$ ) and recorded data from both upper and lower sidebands (USB and LSB). We used 3C273 for our gain, passband, and pointing calibrations. We also observed the quasar J1222+042 and Titan in our 2010 VEX observations for further calibration and its verification. Flux calibration was made using Titan and Ganymede in 2009, and Titan in 2010 and 2012. The angular distances from our gain calibrator 3C273 to NGC 4418, J1222+042, and Titan in our 2010 observations, were only $3.0,2.7$, and 4.0 , respectively. This mitigated calibration errors due to directiondependent phase de-correlation, atmospheric transmission, and pointing offsets.

We reduced our data using MIR for calibration (Scoville et al. 1993), MIRIAD for imaging (Sault et al. 1995), and AIPS (Bridle \& Greisen 1994) and stand-alone programs for further data analyses. The data were first imaged at $30 \mathrm{~km} \mathrm{~s}^{-1}$ resolution for the entire bandwidths to identify lines. Then the "line-free" channels were identified and integrated to create continuum data. That continuum is then subtracted from the original to make continuum-subtracted visibilities. The spectra and the identified lines are presented in Section 3.1. The line data were further analyzed after binning to $10-30 \mathrm{~km} \mathrm{~s}^{-1}$ resolutions. We did self-calibration of phase using the continuum data when making images from the VEX data, although we did not do that when measuring the source position or fitting the visibilities to determine the size and flux density of the continuum source. Table 3 summarizes the properties of the images and spectral data cubes used in this paper.

\section{2. $660 \mathrm{GHz}(450 \mu \mathrm{m})$ Observations}

We also observed the galaxy at $660 \mathrm{GHz}(450 \mu \mathrm{m})$ during our VEX $350 \mathrm{GHz}$ observations in 2009 and 2010 by allocating $2 \mathrm{GHz}$ of correlator bandwidth to our high-frequency receivers. Only the 2010 run resulted in useable data from five antennas. Atmospheric absorption was significant at our observing frequency even though the weather condition was excellent for 

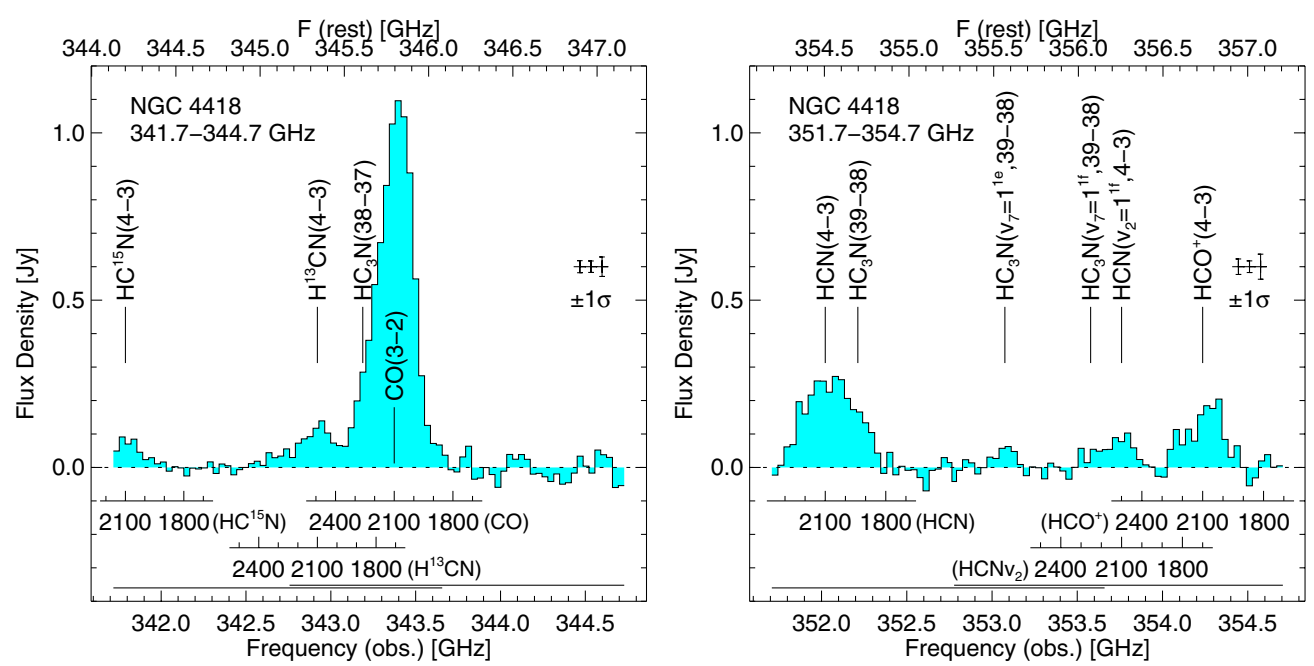

Figure 2. NGC 4418 spectra in the central 0".5 (80 pc; FWHM). Data from the VEX array configuration are used and continuum has been subtracted. Emission lines are marked at the redshift of $2100 \mathrm{~km} \mathrm{~s}^{-1}$ and six of them have velocity axes. The data are from two observations with offset frequency coverages indicated with the two horizontal lines at the bottom. The data at the overlapped frequencies are averaged. The three error bars in each panel are for the three spectral sections (e.g., the middle one for the averaged section).

(A color version of this figure is available in the online journal.)

Table 3

Data Properties

\begin{tabular}{|c|c|c|c|c|c|c|c|}
\hline \multirow{2}{*}{$\begin{array}{l}\text { ID } \\
\text { (1) }\end{array}$} & \multirow{2}{*}{$\begin{array}{c}\text { Data Source } \\
\text { (2) }\end{array}$} & \multirow{2}{*}{$\begin{array}{l}\text { SB. } \\
\text { (3) }\end{array}$} & \multirow{2}{*}{$\begin{array}{l}\text { Emission } \\
\text { (4) }\end{array}$} & \multirow{2}{*}{$\begin{array}{c}\text { Beam } \\
\left("{ }^{\prime \prime}\right) \\
(5)\end{array}$} & \multirow{2}{*}{$\begin{array}{c}\Delta V \\
\left(\mathrm{~km} \mathrm{~s}^{-1}\right) \\
(6)\end{array}$} & \multicolumn{2}{|c|}{ Noise rms } \\
\hline & & & & & & $\begin{array}{c}\left(\mathrm{mJy} \mathrm{beam}^{-1}\right) \\
\text { (7) }\end{array}$ & $\begin{array}{c}\mathrm{K}(\mathrm{R}-\mathrm{J}) \\
(8)\end{array}$ \\
\hline 01 & 1 & $\mathrm{U}$ & $\mathrm{HCO}^{+}(4-3)$ etc. & $0.34 \times 0.24$ & 30 & 27 & 3.2 \\
\hline 02 & 2 & $\mathrm{U}$ & $\mathrm{HCN}(4-3)$ etc. & $0.36 \times 0.26$ & 30 & 20 & 2.2 \\
\hline 03 & $1+2$ & $\mathrm{~L}$ & $\mathrm{CO}(3-2)$ & $0.35 \times 0.26$ & 10 & 22 & 2.4 \\
\hline 04 & $1+2$ & $\mathrm{D}$ & $860 \mu \mathrm{m}$ cont. & $0.35 \times 0.26$ & $\ldots^{\mathrm{a}}$ & 2.4 & 0.27 \\
\hline 05 & $1+3+4$ & $\mathrm{U}$ & $\mathrm{HCO}^{+}(4-3)$ & $0.67 \times 0.45$ & 20 & 15 & 0.47 \\
\hline 06 & $2+3+4$ & $\mathrm{U}$ & $\mathrm{HCN}(4-3)$ & $0.53 \times 0.41$ & 20 & 16 & 0.72 \\
\hline 07 & $1+2+3+4$ & $\mathrm{~L}$ & $\mathrm{CO}(3-2)$ & $0.69 \times 0.55$ & 10 & 14 & 0.38 \\
\hline 08 & $3+4$ & $\mathrm{~L}$ & $\mathrm{CS}(7-6)$ & $0.91 \times 0.59$ & 20 & 16 & 0.32 \\
\hline 09 & $3+4$ & $\mathrm{U}$ & $4 \mathrm{GHz}$ spectrum & $0.92 \times 0.73$ & 30 & 12 & 0.17 \\
\hline 10 & $3+4$ & $\mathrm{~L}$ & $4 \mathrm{GHz}$ spectrum & $1.00 \times 0.76$ & 30 & 11 & 0.15 \\
\hline 11 & $2^{\prime}$ & $\mathrm{D}$ & $450 \mu \mathrm{m}$ cont. & $0.23 \times 0.15$ & $\ldots{ }^{\mathrm{a}}$ & 65 & 5.3 \\
\hline
\end{tabular}

Notes. Column 2: data source. The numbers are those in Table 2. Column 3: sideband. $\mathrm{U}=\mathrm{USB}, \mathrm{L}=\mathrm{LSB}, \mathrm{D}=$ double sideband $=\mathrm{USB}+\mathrm{LSB}$. Column 4: main emission in the data. Column 5: major and minor axes (FWHM) of the synthesized beam. Column 6: velocity resolution. Columns 7 and 8: noise rms intensity in the data. For lines this is for a channel of the width in Column 6. The noise in Column 8 is in K and in Rayleigh-Jeans brightness temperature.

${ }^{a}$ The mean frequency and total bandwidth of the continuum are, respectively, 348.2 and $3.5 \mathrm{GHz}$ for the $860 \mu \mathrm{m}$ data and $663.0 \mathrm{and} 3.9 \mathrm{GHz}$ for the $450 \mu \mathrm{m}$ data.

Mauna Kea. The mean zenith opacity was 0.038 at $225 \mathrm{GHz}$ and 0.89 at $350 \mu \mathrm{m}$ during our observations according to the monitoring at the adjacent Caltech Submillimeter Observatory. The opacity should be about the same at 450 and $350 \mu \mathrm{m}$. The median double sideband (DSB) system temperature was about $1200 \mathrm{~K}$ toward NGC 4418 . We used 3C273 for both passband and gain calibration. Both 350 and $660 \mathrm{GHz}$ data were used for the gain calibration. We first applied the $350 \mathrm{GHz}$ gain solution with its phase multiplied by the ratio of the observing frequencies, 1.9, and then derived another gain solution using the $660 \mathrm{GHz}$ data and applied it to improve the calibration. No strong line is in our passband and the data are treated as continuum. Since Titan is too heavily resolved, flux calibration at $660 \mathrm{GHz}$ is based on the $350 \mathrm{GHz}$ flux density of 3C273 scaled to $660 \mathrm{GHz}$ using the spectral index of $\alpha=-1.0$ (S10). We estimate our flux measurements at $660 \mathrm{GHz}$ to be accurate only to $36 \%$ (for $\sigma(\alpha)=0.3$ and $30 \%$ error for other calibrations).

\section{OBSERVATIONAL RESULTS AND ANALYSIS}

\subsection{Spectra and Line-Continuum Separation}

Figures 2 and 3 show spectra ${ }^{7}$ made from the VEX data alone and from the EXT and SC data, respectively. The SC + EXT data are two times more sensitive than the SC data alone reported in S10.

Two lines among about 10 in the spectra are new detections in this galaxy. Both are from vibrationally excited $\mathrm{HC}_{3} \mathrm{~N}$. Namely, $\mathrm{HC}_{3} \mathrm{~N}\left(v_{7}=1^{1 f}, J=39-38\right)$ is at $f_{\text {rest }}=356.072 \mathrm{GHz}$ and has its upper energy level at $E_{\mathrm{u}}=663 \mathrm{~K} . \mathrm{HC}_{3} \mathrm{~N}\left(v_{6}=1^{1 e}, J=\right.$

\footnotetext{
7 Throughout this paper, velocities are with respect to the local standard of rest (LSR) and are defined with the radio convention, i.e.,

$v_{\text {radio }}=c\left(1-\left(f_{\text {obs }} / f_{\text {rest }}\right)\right)$ where $f$ denotes frequency and $c$ the speed of light. The conversion to velocity in the optical definition is $v_{\text {opt }}=c z=v_{\text {radio }}+15 \mathrm{~km} \mathrm{~s}^{-1}$ for NGC 4418 .
} 

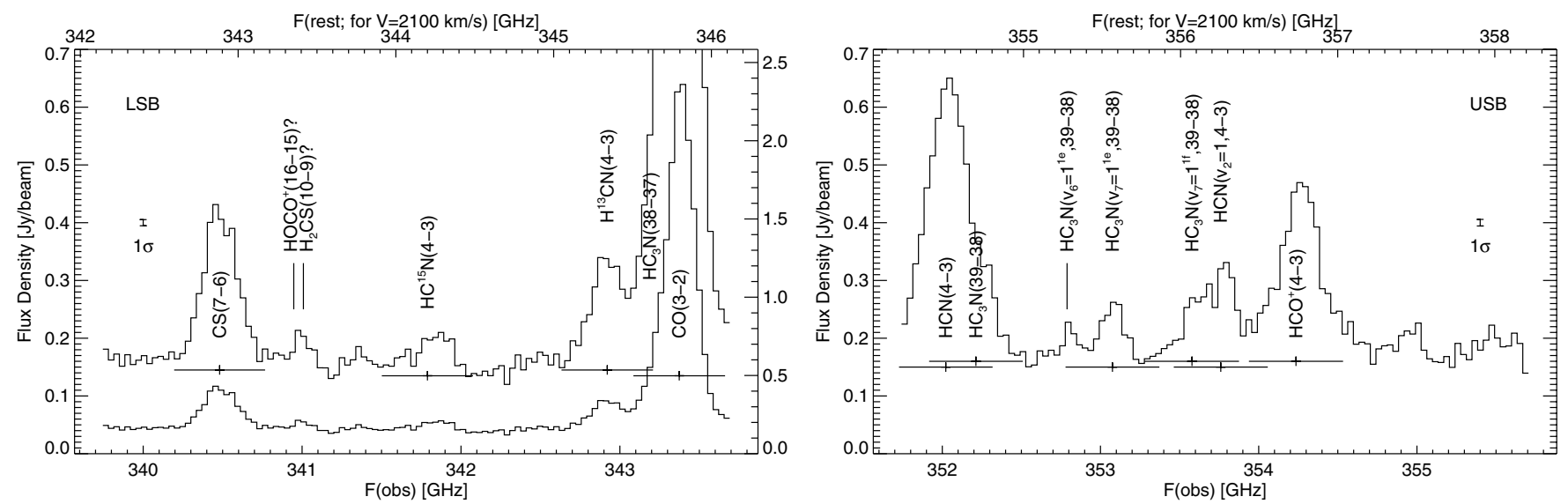

Figure 3. NGC 4418 spectra in the central $\sim 1^{\prime \prime}$ (data ID 09 and 10 in Table 3). No continuum subtraction has been made. The same spectrum is plotted twice for the LSB data to show weak features (left scale) and the full spectrum (right scale). Horizontal bars under strong lines indicate the velocity ranges of $1850-2350 \mathrm{~km} \mathrm{~s}^{-1}$, which are excluded when making continuum data. The central tick of each bar is at $2100 \mathrm{~km} \mathrm{~s}^{-1}$.

$39-38)$ is at $f_{\text {rest }}=355.278 \mathrm{GHz}$ and has $E_{\mathrm{u}}=1059 \mathrm{~K}$. The doublet counterpart of the former line $\left(v_{7}=1^{1 e}, J=39-38\right)$ was already identified at $f_{\text {rest }}=355.566 \mathrm{GHz}$ in S10. Lower $J$ transitions of the latter line were detected in Costagliola \& Aalto (2010) and C12. Its doublet counterpart $\left(v_{6}=1^{1 f}, J=39-38\right)$ at $f_{\text {rest }}=355.557 \mathrm{GHz}$ is blended with the $\mathrm{HC}_{3} \mathrm{~N}\left(v_{7}=1^{1 e}, J=\right.$ 39-38) mentioned above. Although unidentified, there may be more lines, e.g., at $f_{\text {rest }} \sim 343.4$ and $357.5 \mathrm{GHz}$; species having a line at the former frequency include $\mathrm{H}_{2} \mathrm{CS}$ and $\mathrm{HOCO}^{+}$.

The $350 \mathrm{GHz}$ continuum in each sideband was made by averaging the following channels. For the VEX data we used channels outside of 1900-2300 $\mathrm{km} \mathrm{s}^{-1}$ for $\mathrm{CO}(3-2)$, $\mathrm{HCN}(4-3), \mathrm{H}^{13} \mathrm{CN}(4-3), \mathrm{HC}^{15} \mathrm{~N}(4-3), \mathrm{HCN}\left(v_{2}=1^{1 f}, J=\right.$ 4-3), $\mathrm{HCO}^{+}(4-3), \mathrm{CS}(7-6)$, and $\mathrm{HC}_{3} \mathrm{~N}(39-38)$ and (38-37). For the EXT and SC data we excluded channels having velocities of $1850-2350 \mathrm{~km} \mathrm{~s}^{-1}$ for the same lines ${ }^{8}$ plus $\mathrm{HC}_{3} \mathrm{~N}\left(v_{7}=1^{1 f}, J=\right.$ 39-38) and $\left(v_{7}=1^{1 e}, J=39-38\right)$, channels between $\mathrm{CO}(3-2)$ and its nearest band edge, and the same for $\operatorname{HCN}(4-3)$. The $660 \mathrm{GHz}$ continuum was averaged over the full bandwidth and both sidebands.

\subsection{Concentration of Gas and Dust in the Nucleus}

Submillimeter emission is detected only from the central kpc $\left(\sim 6^{\prime \prime}\right)$ with different spatial extents in various emission components. We show this below using maps, aperture photometry, and visibility fitting.

\subsubsection{Maps}

Figure 4 shows $\mathrm{CO}(3-2)$ channel maps to display faint extended emission at 0 .'6 resolution. The data cube was made by combining all our data. We detected here $797 \pm 80 \mathrm{Jy} \mathrm{km} \mathrm{s}^{-1}$ or $84 \% \pm 18 \%$ of the single-dish $\mathrm{CO}(3-2)$ flux observed in the central 14 " of the galaxy by Yao et al. (2003). Despite the high recovery rate, the $\mathrm{CO}(3-2)$ emission was detected only in the central $6^{\prime \prime}(1 \mathrm{kpc})$ of the galaxy, showing that the extent of the $\mathrm{CO}(3-2)$ emission is intrinsically smaller than our $34^{\prime \prime}(5.6 \mathrm{kpc})$ field of view. The $\mathrm{CO}$ emission is not only compact but also strongly peaked toward the nucleus within the central kpc. In this data set more than half $(58 \%)$ of the $\mathrm{CO}(3-2)$ flux in the central kpc is in the central $165 \mathrm{pc}\left(1^{\prime \prime}, \mathrm{FWHM}\right)$.

\footnotetext{
8 The velocity range used here is the same as the one used to analyze the SC
} data in S10 although the note in their Table 1 described it otherwise.
Figures 5 and 6, respectively, are $\mathrm{CO}(3-2)$ and $\mathrm{HCN}(4-3)$ channel maps at $\sim 0$ '.3 (50 pc) resolutions showing that the line emission is strongly peaked at the nucleus at subarcsec $(\lesssim 100$ pc) scales. These data cubes were made from the VEX data alone. This $\mathrm{CO}(3-2)$ data cube contains about $50 \%$ of the $\mathrm{CO}$ flux detected in our 0.6 resolution data.

Our continuum and line moment maps are shown in Figures 7 and 8. The spatial resolutions are about $0^{\prime \prime} .6$ and $0^{\prime \prime} .3$ for $350 \mathrm{GHz}$ data and 0.2 for $660 \mathrm{GHz}$ continuum. In particular, Figures $8(\mathrm{k})$ and (l) show our detection of a compact continuum source at the galactic center. The continuum position is listed in Table 1 and agrees to $\sim 0.3$ with the optical and radio positions of the nucleus; see the caption of Table 1 for the optical position and Condon et al. (1990) for the radio position.

\subsubsection{Aperture Photometry}

The degree of central concentration, or compactness, is compared among various lines and continuum at $350 \mathrm{GHz}$ in Table 4 using the ratio of flux (or flux density) between the central $0.5(80 \mathrm{pc})$ and $66^{\prime \prime}(1 \mathrm{kpc})$. The former photometry is made using our data from the VEX configuration and the latter data are from S10 that used the SC configuration. The ratios for various emission components indicate their relative compactness. The relatively extended nature of $\mathrm{CO}(3-2)$ emission is evident from its low ratio. Only one-third of the $\mathrm{CO}(3-2)$ emission is from the central 0.'5. Lines other than $\mathrm{CO}$ have higher flux ratios, and hence are more concentrated toward the nucleus. The ratio for $860 \mu \mathrm{m}$ continuum, $0.95 \pm 0.07$, is the highest among the ratios with the signal-to-noise ratio $(\mathrm{S} / \mathrm{N})>3$ in Table 4 . Thus, $860 \mu \mathrm{m}$ continuum emission is more compact than both the $\mathrm{CO}$ and non-CO high excitation lines. At the same time, the continuum emission in the VEX data is slightly more extended than the 0.3 synthesized beam as seen in the last two panels of Figure 5. The detected $450 \mu \mathrm{m}$ continuum also looks compact but it has only a minor fraction of single-dish measurement.

\subsubsection{Visibility Fitting: $860 \mu \mathrm{m}$ Continuum}

Figures 9(a)-(c) show fits to the continuum visibilities of NGC 4418, a test point source $\mathrm{J} 1222+042$, and Titan whose subarcsec size is known. The fit results are listed in Table 5. For NGC 4418, data from the three array configurations are combined. This cannot be done for the calibrators because 


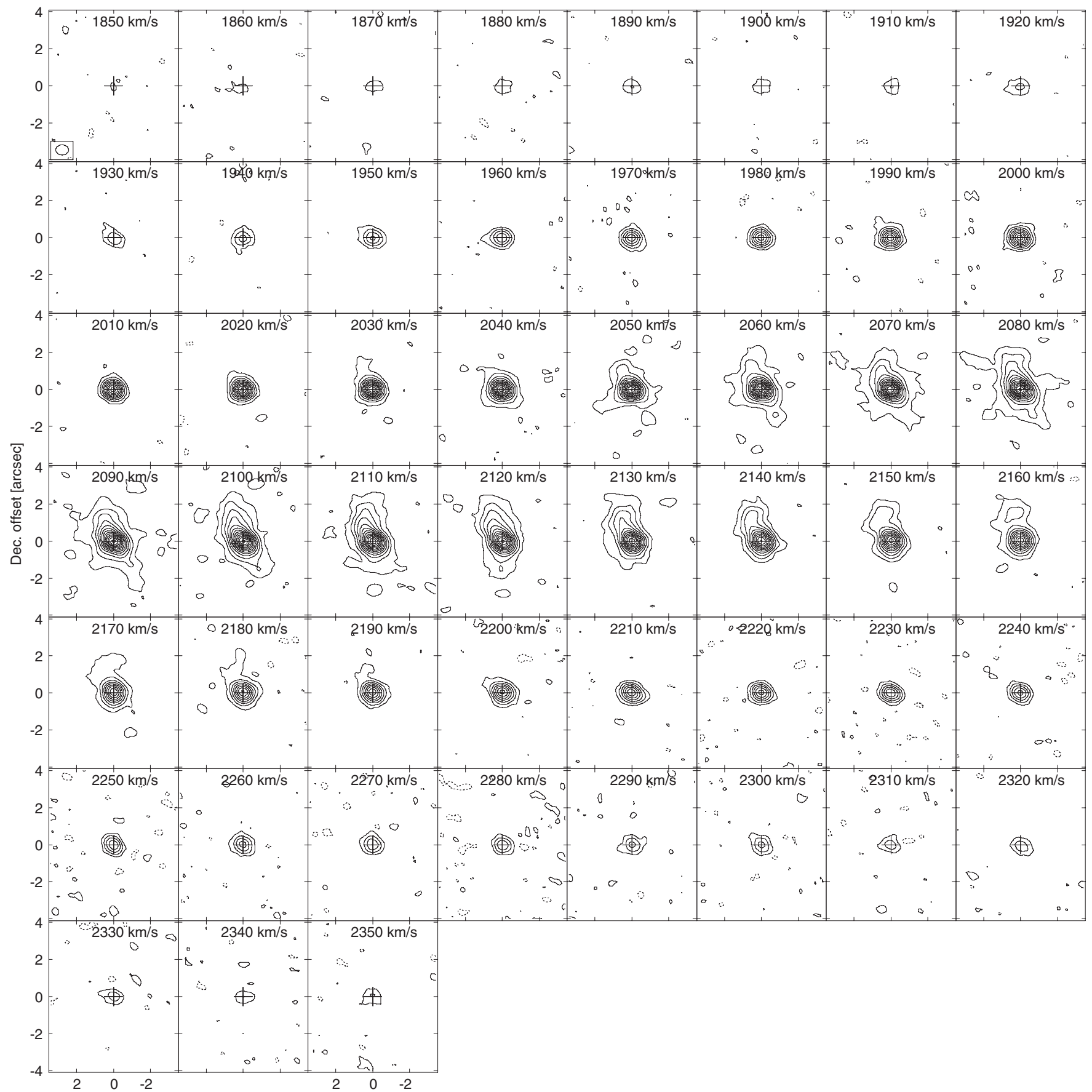

R.A. offset [arcsec]

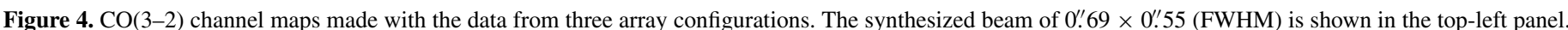

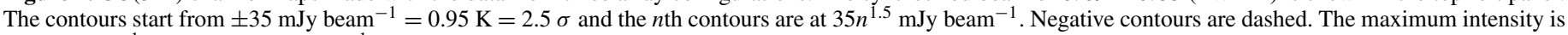

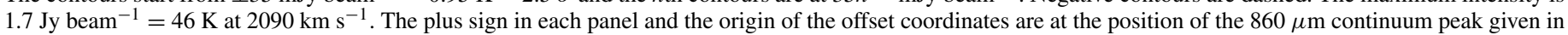
Table 1.

their flux densities and the apparent diameter of Titan change with time. Since the NGC 4418 data were taken in three different nights the three data sets should have independent flux calibration errors, which are typically about $10 \%-20 \%$ for the SMA $350 \mathrm{GHz}$ observations. However, we did not add the flux scaling error in the plot and in our fitting because doing so would make the reduced $\chi^{2}$ well below unity. It is most likely that we achieved better flux calibration than usual, presumably because we had accurate pointing from repeated pointing on the bright quasar 3C273 next to NGC 4418, our primary flux calibrator Titan was also close to $3 \mathrm{C} 273$ and NGC 4418, and atmospheric absorption was low $(225 \mathrm{GHz}$ zenith opacity $\leqslant 0.05$ for all the three nights). The size of $850 \mu \mathrm{m}$ continuum emission of NGC 4418 obtained from our data is 0 ". 10 (FWHM). We will use this for the source modeling in Section 3.3.

We verified our visibility fitting using the quasar J1222+042 and Titan. They were gain calibrated using 3C273 in the same way as NGC 4418 was calibrated. Our fit of J1222+042 using a Gaussian shows that blurring of the point source was less than 50 mas in our observations. The diameter of Titan at $860 \mu \mathrm{m}$ is known to be $5230 \mathrm{~km}$ or 0.8425 for the distance of 8.5588 AU. This size includes the $40 \mathrm{~km}$ altitude of the tropopause, around 


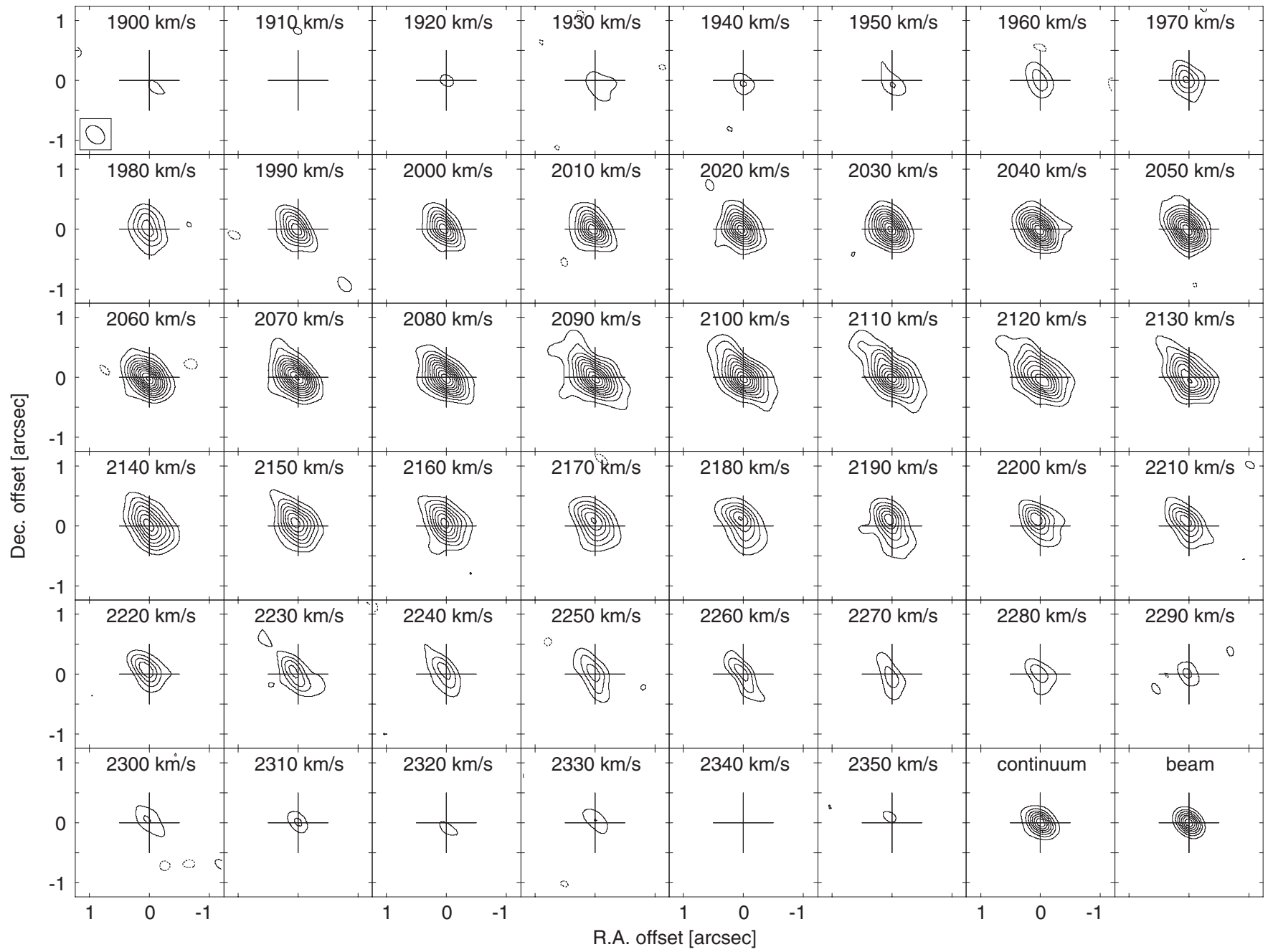

Figure 5. $\mathrm{CO}(3-2)$ channel maps at the center of NGC 4418. Only the data from the VEX configuration are used. Contours are at $-3 \sigma, 3 \sigma, 6 \sigma, 9 \sigma, 12 \sigma, \ldots, 33 \sigma$, where $3 \sigma=65 \mathrm{mJy}_{\text {beam }}^{-1}=7.3 \mathrm{~K}$. Negative contours are dashed. The maximum intensity is $730 \mathrm{mJy} \mathrm{beam}^{-1}=82 \mathrm{~K}$ at 2060 and $2070 \mathrm{~km} \mathrm{~s}^{-1}$. The plus sign in each panel and the origin of the offset coordinates are at the position of the $860 \mu \mathrm{m}$ continuum peak given in Table 1 . The synthesized beam of 0 '! $35 \times 0$ '.26 (FWHM) is shown in the top-left panel. The last two panels show LSB continuum and the point-spread function (i.e., CLEAN beam), respectively, in contour steps of $12.5 \%$ of each peak.

Table 4

Peak Brightness Temperatures and Fluxes in the VEX Data

\begin{tabular}{|c|c|c|c|c|}
\hline \multirow{2}{*}{$\begin{array}{l}\text { Emission } \\
\text { (1) }\end{array}$} & \multicolumn{2}{|c|}{$\operatorname{Max} T_{\mathrm{b}}$} & \multirow{2}{*}{$\begin{array}{c}S, S_{\lambda}\left(0^{\prime \prime} 5 \text { FWHM }\right) \\
\left(\mathrm{Jy} \mathrm{km} \mathrm{s}^{-1}, \mathrm{mJy}\right) \\
(4)\end{array}$} & \multirow{2}{*}{$\begin{array}{c}f\left(0^{\prime \prime} 5 / 6^{\prime \prime}\right) \\
(5)\end{array}$} \\
\hline & $\begin{array}{c}\mathrm{K}(\mathrm{R}-\mathrm{J}) \\
(2)\end{array}$ & $\begin{array}{c}\mathrm{K} \text { (Planck) } \\
\text { (3) }\end{array}$ & & \\
\hline $\mathrm{CO}(J=3-2)$ & 82 & 90 & $240 \pm 3$ & $0.32 \pm 0.02$ \\
\hline $\operatorname{HCN}(J=4-3)$ & 24 & 31 & $80 \pm 3$ & $0.60 \pm 0.05$ \\
\hline $\mathrm{HCO}^{+}(J=4-3)$ & 20 & 28 & $39 \pm 4$ & $0.48 \pm 0.06$ \\
\hline $\mathrm{H}^{13} \mathrm{CN}(J=4-3)$ & 13 & 20 & $29 \pm 4$ & $0.71 \pm 0.13$ \\
\hline $\mathrm{HC}^{15} \mathrm{~N}(J=4-3)$ & 8 & 15 & $17 \pm 4$ & $1.55 \pm 0.57$ \\
\hline $\operatorname{HCN}\left(v_{2}=1^{1 f}, J=4-3\right)$ & 13 & 20 & $18 \pm 4$ & $0.60 \pm 0.15$ \\
\hline $860 \mu \mathrm{m}$ continuum & 17 & 24 & $168 \pm 3$ & $0.95 \pm 0.07$ \\
\hline $450 \mu \mathrm{m}$ continuum & 31 & 45 & $369 \pm 37$ & $\ldots$ \\
\hline
\end{tabular}

Notes. Columns 2 and 3: peak brightness temperatures measured in the VEX data (data ID 01-04 and 11 in Table 3). No correction is made for missing flux and beam dilution. Temperatures calculated using the Rayleigh-Jeans approximation and the Planck function are given in Columns 2 and 3, respectively. The max $T_{\mathrm{b}}$ is measured in the $10 \mathrm{~km} \mathrm{~s}^{-1}$ resolution data for $\mathrm{CO}$ and $30 \mathrm{~km} \mathrm{~s}^{-1}$ for other lines. Column 4: line fluxes and continuum flux densities that we detected in the central 0!'5 (FWHM). They were measured from data ID 01-04 and 11 in Table 3 after convolving them to the resolution. The line fluxes are integrated over $1900-2300 \mathrm{~km} \mathrm{~s}^{-1}$; for $\mathrm{H}^{13} \mathrm{CN}$ and $\mathrm{HC}^{15} \mathrm{~N}$ we doubled the flux of half that width since the other half of the line is either outside of our bandwidth or blended with another line. Note that the CO, $\mathrm{HCN}$, and $\mathrm{HCN}\left(v_{2}=1\right)$ lines are blended with a nearby $\mathrm{HC}_{3} \mathrm{~N}$ transition. The $1 \sigma$ errors are for the thermal noise. We estimate additional flux-scaling error to be $\sim 5 \%$ for $345 \mathrm{GHz}$ data and $40 \%$ for $660 \mathrm{GHz}$ data. Column 5: fraction of flux detected in our $0^{\prime \prime} 5$ data compared to the $6^{\prime \prime}$ resolution data in S10. 


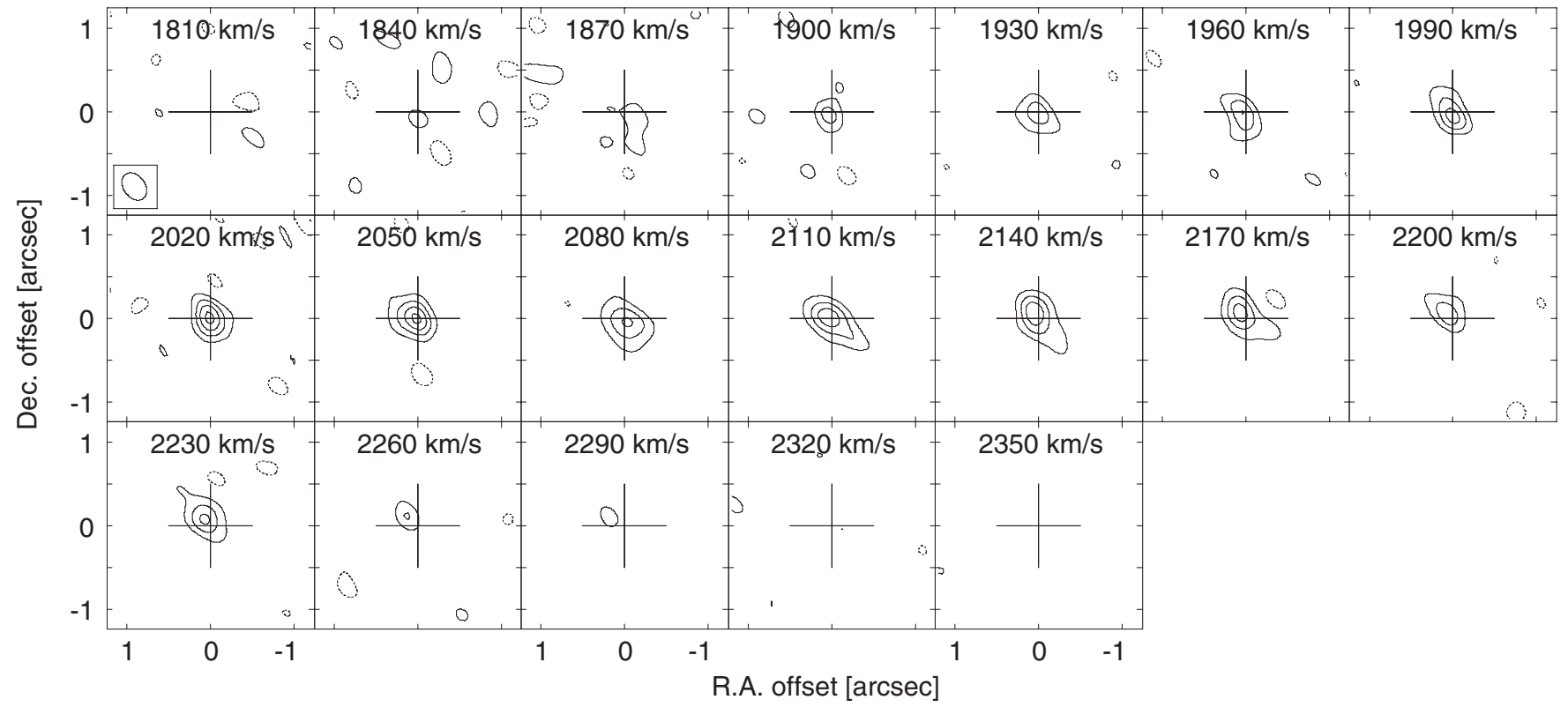

Figure 6. HCN(4-3) channel maps at the center of NGC 4418. Only the data from the VEX configuration are used. Contours are at $(-1,1,2,3,4) \times\left(50 \mathrm{mJy}\right.$ beam ${ }^{-1}=$ $5.4 \mathrm{~K}=2.5 \sigma)$. Negative contours are dashed. The maximum intensity is $23 \mathrm{~K}$ at 2020 and $2050 \mathrm{~km} \mathrm{~s}^{-1}$. The plus sign in each panel and the origin of the offset coordinates are at the position of the $860 \mu \mathrm{m}$ continuum peak given in Table 1. The synthesized beam of $0{ }^{\prime} 36 \times 0$ '”26 (FWHM) is shown in the top-left panel.
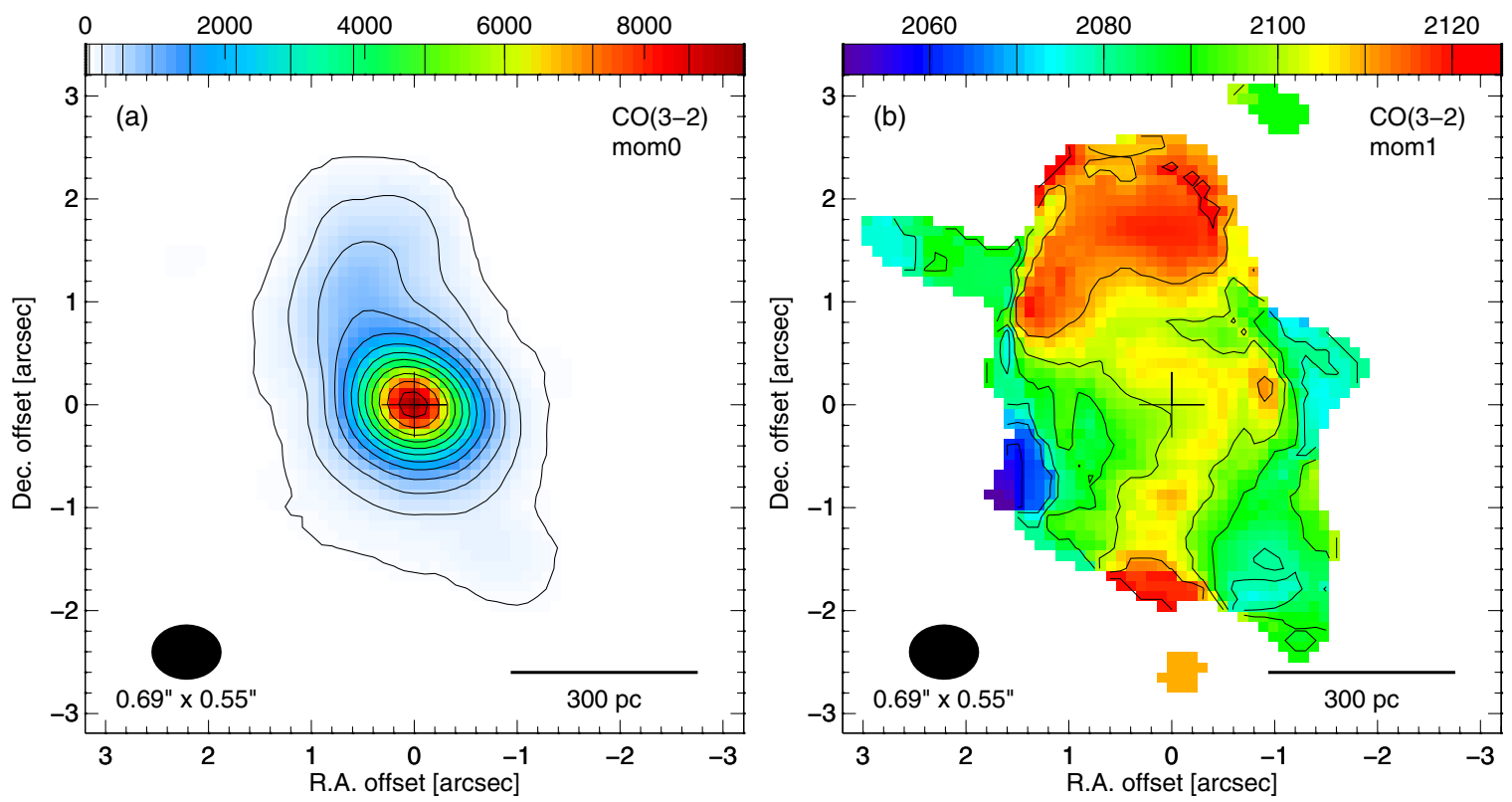

Figure 7. $\mathrm{CO}(3-2)$ maps made using three array configurations. (a) Integrated intensity. The $n$th contour is at $60 n^{2} \mathrm{~K} \mathrm{~km} \mathrm{~s}^{-1}$. The peak intensity is $9.4 \times 10^{3} \mathrm{~K} \mathrm{~km} \mathrm{~s}{ }^{-1}$. (b) Intensity-weighted mean velocity. Contour interval is $10 \mathrm{~km} \mathrm{~s}^{-1}$. The plus sign in each panel and the origin of the offset coordinates are at the position of the $860 \mu \mathrm{m}$ continuum peak given in Table 1 . The black ellipses show the FWHM of the synthesized beam.

(A color version of this figure is available in the online journal.)

which is the submillimeter photosphere of Titan. The distance at the time of our 2010 VEX observations is from JPL-Horizons. Figure 9(c) shows that our data match the expected visibility curve very well with only flux scaling. When the data were fit for both the source size and amplitude scaling, we obtained the size of the subarcsec source with an accuracy better than $3 \%$ or to 20 mas.

The flux density of Titan sets the absolute amplitude scaling of all the visibility fitting. We adopt the continuum brightness temperature of $77 \mathrm{~K}$ as the Planck temperature that gives the total flux density of Titan for the solid-surface diameter $(5150 \mathrm{~km})$ of the satellite (M. Gurwell 2010, private communication). Our
Titan data do not contain the $\mathrm{CO}(3-2)$ and $\mathrm{HCN}(4-3)$ lines, both of which are known to be bright. Still, our imperfect knowledge of the atmosphere of Titan may introduce a common amplitude error to our data even though the relative calibration using Titan is precise and consistent among the three data sets. We therefore assign $5 \%$ to the overall flux scaling uncertainty of our NGC 4418 fit, even though the formal error in the fitting was about $1 \%$. In addition to this, there is likely contamination of line emission to the "line-free" channels used for the USB continuum of the galaxy. We observed a sign of that in our SC configuration data in the form of an uncomfortably large spectral index $\alpha=6.25 \pm 0.84\left(S_{v} \propto v^{\alpha}\right)$ between the two sidebands 

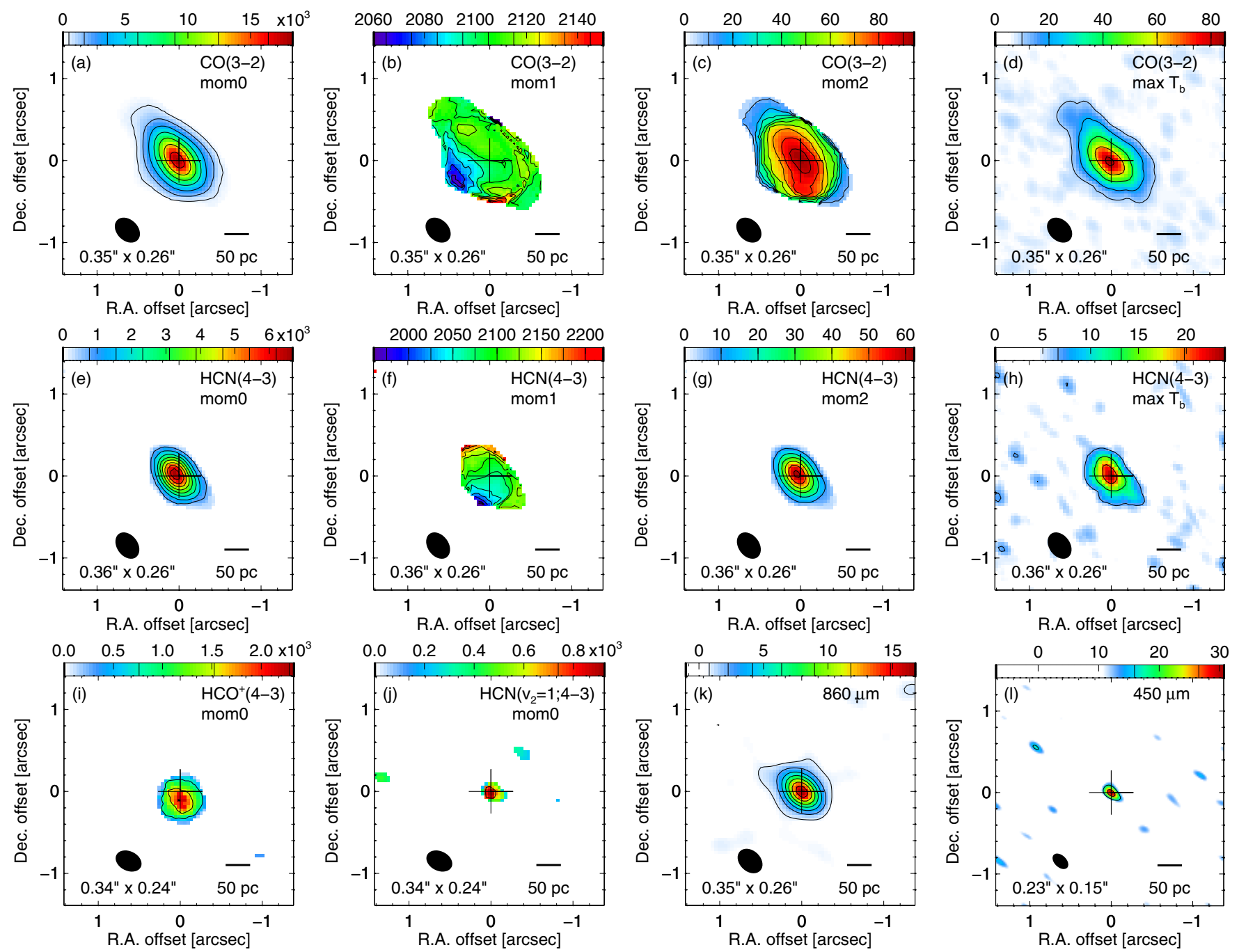

Figure 8. Submillimeter line and continuum images at the center of NGC 4418; (a)-(d) are $\mathrm{CO}(3-2)$, (e)-(h) are $\mathrm{HCN}(4-3)$, (i) is $\mathrm{HCO}^{+}(4-3)$, (j) is $\mathrm{HCN}\left(v_{2}=1^{1 f}\right.$, $J=4-3$ ), and (k) and (1) are 860 and $450 \mu \mathrm{m}$ (DSB) continuum, respectively. Data from the VEX configuration are used. The position of the $860 \mu \mathrm{m}$ continuum peak, which is given in Table 1, is marked with the plus sign in each panel and is used as the origin of the offset coordinates. The black ellipse in each panel shows the FWHM of the synthesized beam. The images labeled with "mom0," "mom1," and "mom2" show integrated intensity (i.e., zeroth moment) in $\mathrm{K} \mathrm{km} \mathrm{s}{ }^{-1}$, intensity-weighted mean velocity (first moment) in $\mathrm{km} \mathrm{s}^{-1}$, and velocity dispersion (second moment) in $\mathrm{km} \mathrm{s}^{-1}$, respectively, and those labeled with "max $T_{\mathrm{b}}$ " show peak brightness temperature in $\mathrm{K}$. The contour information is as follows. (a) The $n$th contour is at $0.5 n^{1.8} \times 10^{3} \mathrm{~K} \mathrm{~km} \mathrm{~s}^{-1}$. The peak value is $18 \times 10^{3} \mathrm{~K} \mathrm{~km} \mathrm{~s}^{-1}$. (b) $10 \mathrm{~km} \mathrm{~s}-1$ steps. The contour crossing the continuum peak is at $2105 \mathrm{~km} \mathrm{~s}^{-1}$. (c) $10 \mathrm{~km} \mathrm{~s}^{-1}$ steps. (d) $10,20,40,60$, and $80 \mathrm{~K}(1 \sigma=2.4 \mathrm{~K})$. The peak is $82 \mathrm{~K}$. (e) $0.78 n 10^{3} \mathrm{~K} \mathrm{~km} \mathrm{~s}$. The peak is $6.7 \times 10^{3} \mathrm{~K} \mathrm{~km} \mathrm{~s}^{-1}$. (f) $25 \mathrm{~km} \mathrm{~s}^{-1}$ steps. The contour crossing the continuum peak is $2075 \mathrm{~km} \mathrm{~s}^{-1}$. (g) $10 \mathrm{~km} \mathrm{~s}^{-1}$ steps. (h) $(1,2,3) \times(6.6 \mathrm{~K}=3 \sigma)$. The peak is $23 \mathrm{~K}$. (i) $(1,2,3) \times 0.76 \times 10^{3} \mathrm{~K} \mathrm{~km} \mathrm{~s}^{-1}$. (j) The contour is at $0.74 \times 10^{3} \mathrm{~K} \mathrm{~km} \mathrm{~s}^{-1}$. (k) $0.8 n^{1.5} \mathrm{~K}$, starting at $3 \sigma$. The intensity peak is $17 \mathrm{~K}$. (1) Contours are at $(-3,3,5) \times\left(1 \sigma=5.3 \mathrm{~K}=65 \mathrm{mJy}\right.$ beam $\left.^{-1}\right)$. Peak $=31 \mathrm{~K}(\mathrm{R}-\mathrm{J})=374 \mathrm{mJy}^{\mathrm{beam}}{ }^{-1}=5.8 \sigma$.

(A color version of this figure is available in the online journal.)

(S10). A similar sign was seen in our 2010 VEX data too. Since we used the USB continuum in the multi-configuration fitting as LSB data were noisier, the line contamination is likely in the fitted amplitude. Its effect on the luminosity estimate is addressed in Section 3.3.3.

\subsubsection{Visibility Fitting: Lines}

Figure 9 also shows visibility amplitudes of line emission against baseline lengths. Line visibilities were averaged over $1900-2300 \mathrm{~km} \mathrm{~s}^{-1}$ after continuum subtraction, and then binned according to the baseline length. It is evident in the figure that the line visibility amplitudes decline faster than the continuum amplitudes. In other words, line emission is more extended than continuum. Comparison of $\mathrm{CO}, \mathrm{HCN}$, and $\mathrm{HCO}^{+}$data tells us that the $\mathrm{CO}$ emission is more extended than the latter two, confirming our observation using the aperture photometry.
Moreover, the $\mathrm{CO}$ visibility plot shows that the data cannot be fit with a single Gaussian in the $u-v$ domain. Hence the spatial distribution of the $\mathrm{CO}(3-2)$ emission is not a Gaussian. The line emission distribution has at least a compact subarcsec peak toward the nucleus (Figure 8) and an extended component with the total extent of $\sim 5^{\prime \prime}$ (Figure 7). The extended component is non-axisymmetric around the nucleus (Figure 7).

\subsection{Parameters of the Continuum Core}

\subsubsection{Spectral Energy Distribution, Continuum Opacity}

The continuum SED has a power-law slope of $\alpha=2.55 \pm$ $0.18\left(S_{\nu} \propto \nu^{\alpha}\right)$ between $1.3 \mathrm{~mm}$ and $0.85 \mathrm{~mm}$. This is measured from the SMA data in Figure 10, namely from this work, S10, and the 1.3 and $1.1 \mathrm{~mm}$ observations used in the companion paper (C12). Since the SMA continuum data were taken from 

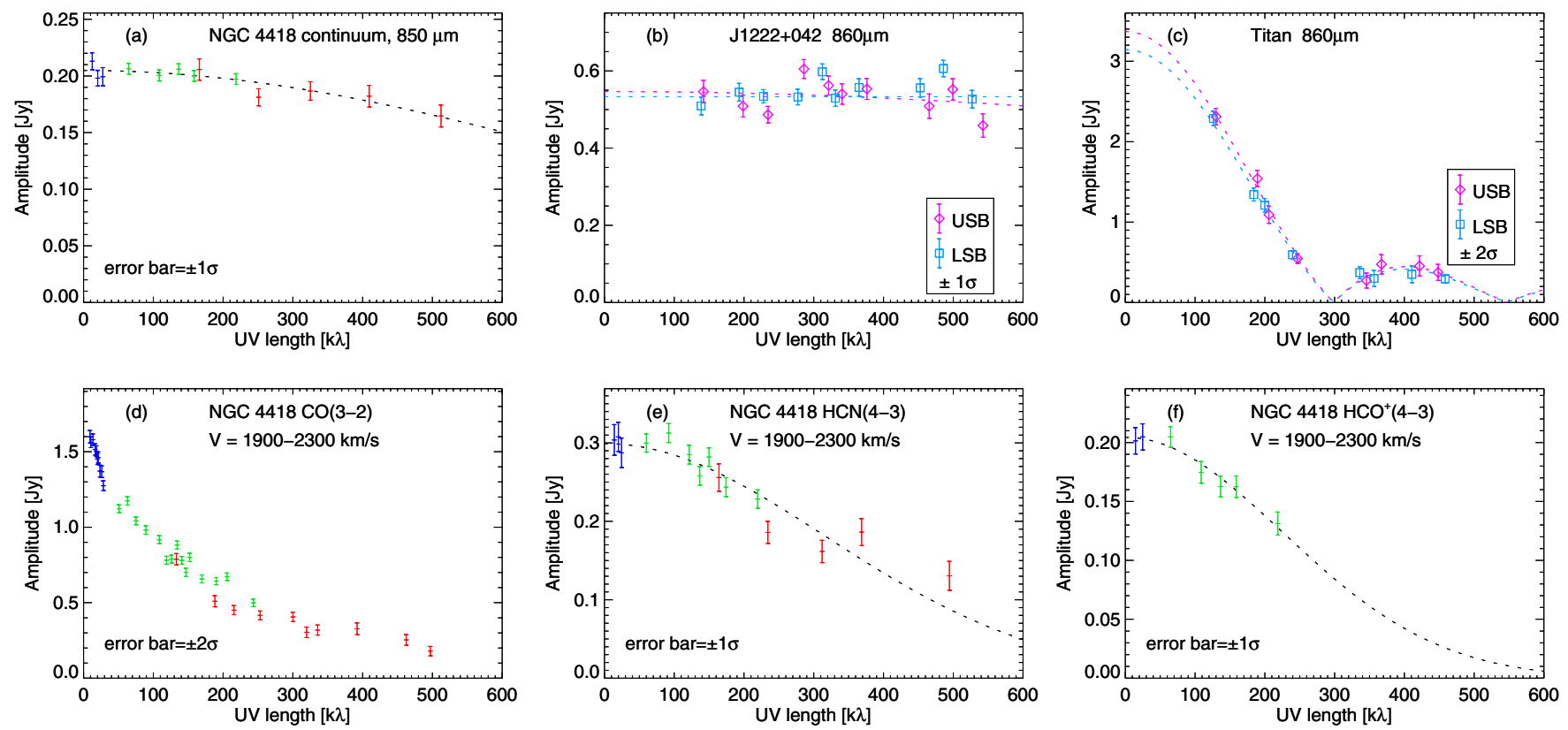

Figure 9. Visibility fitting results. (a)-(c): continuum data of NGC 4418, J1222+042 (a test point source), and Titan (a source with known size). The latter two show 2010 VEX data alone. Dotted lines are Gaussian fits for (a) and (b) and a fit with a circular disk of known diameter for (c). (d)-(e): line data of NGC 4418 integrated over 1900-2300 $\mathrm{km} \mathrm{s}^{-1}$ after continuum subtraction. Data shown in blue, green, and red are from the SC, EXT, and VEX configurations of the SMA, respectively. Dotted lines are models fitted to the data. See Table 5 for the fit parameters. The CO data could not be reasonably fit with a single Gaussian.

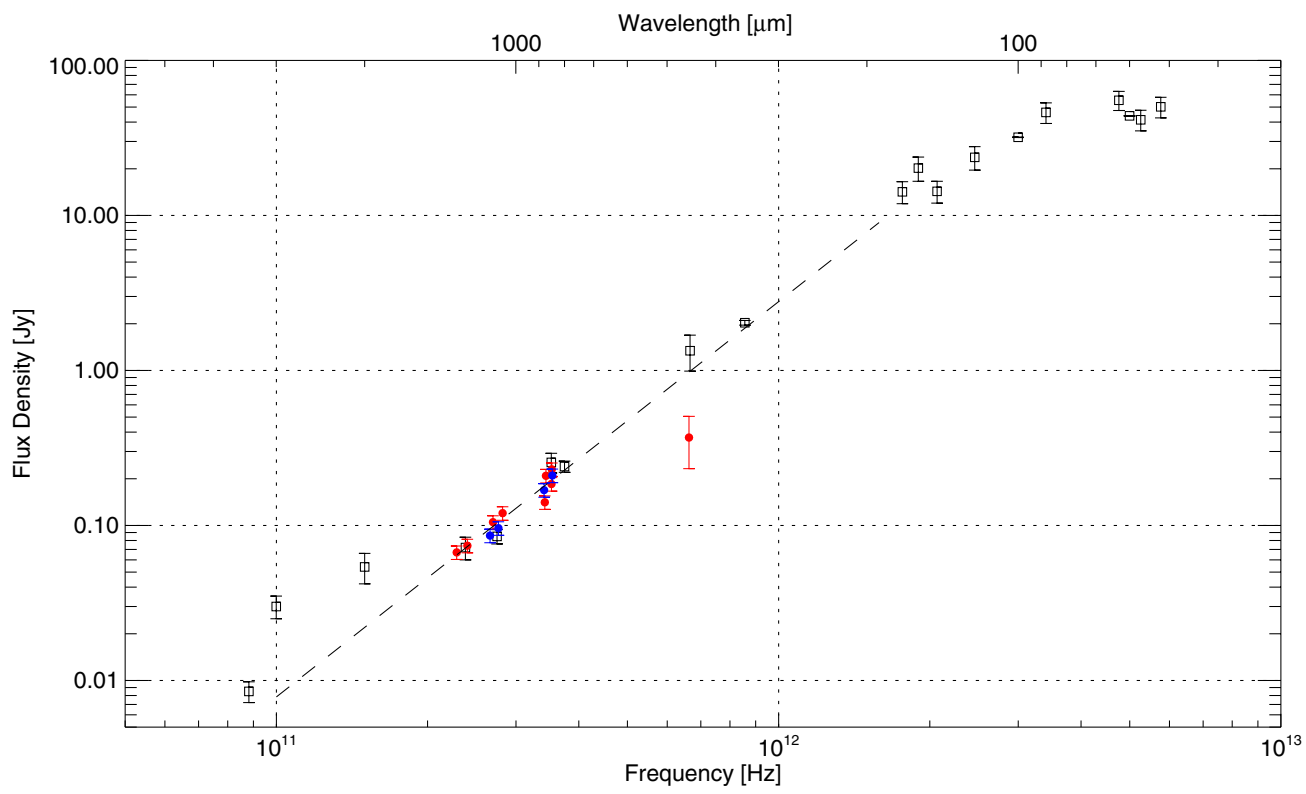

Figure 10. Spectral energy distribution of NGC 4418 in its Rayleigh-Jeans part. Filled data points are from SMA observations; blue ones are from the short baseline observations in Sakamoto et al. (2010) and red ones are from long baseline observations. Error bars are $\pm 1 \sigma$. The dashed line is a power-law fit to the SMA data between 200 and $400 \mathrm{GHz}$. It has a spectral index of $\alpha=2.55 \pm 0.18$ (for $S_{\nu} \propto \nu^{\alpha}$ ) and is extrapolated to a wider frequency range to guide eyes. Non-SMA data are from the literature (Brauher et al. 2008; Sanders et al. 2003; Yang \& Phillips 2007; Roche \& Chandler 1993; Dunne et al. 2000; Imanishi et al. 2004). No subtraction for line contamination is made for the bolometer data between $100 \mathrm{GHz}$ and $1 \mathrm{THz}$.

line-free spectrometer channels in each data set, none of the SMA data is contaminated by strong emission lines such as $\mathrm{CO}$ unlike wide-band bolometer data. The spectral slope that we obtained is shallower (i.e., $\alpha$ is smaller) than those of other local IR luminous galaxies but is consistent with the results of the FIR-to-mm SED surveys of Lisenfeld et al. (2000) and Yang \& Phillips (2007), in each of which NGC 4418 has the shallowest spectral slope in about 15 galaxies.

Two simple models for the spectral index suggest that the $\alpha$ measured at the nucleus is due to the dust opacity $\tau \sim 1$ at $\lambda \sim 1 \mathrm{~mm}$. In the first model of a simple one-zone slab, the spectral slope should be $\alpha=2+\beta \tau\left(e^{\tau}-1\right)^{-1}$, where $\beta$ is the index of power-law frequency dependence of the dust opacity and $\tau$ is the slab opacity at the wavelength of the $\alpha$ measurement. Here we used the Rayleigh-Jeans (R-J) approximation because the brightness temperature of the 0.1 nucleus exceeds $100 \mathrm{~K}$, as we will see next (Section 3.3.3). With optically thin emission, this model gives the familiar form of $\alpha=2+\beta$. The low $\alpha$ observed in NGC 4418 means low $\beta$ or a moderate opacity of dust emission, i.e., $\tau \sim 1$ at $\lambda \sim 1 \mathrm{~mm}$. 
Table 5

Visibility Fitting Results

\begin{tabular}{lcccc}
\hline \hline Source & $\begin{array}{c}\lambda, \text { Line } \\
(\mathrm{mm})\end{array}$ & $\begin{array}{c}\text { Flux Density } \\
(\mathrm{Jy})\end{array}$ & $\begin{array}{c}\text { Size } \\
(\operatorname{arcsec})\end{array}$ & Reduced $\chi^{2}$ \\
$(1)$ & $(2)$ & $(3)$ & $(4)$ & $(5)$ \\
\hline NGC 4418 & 0.85 & $0.205 \pm 0.002$ & $0.101 \pm 0.011$ & 0.63 \\
NGC 4418 & $\mathrm{HCN}^{+4-3)}$ & $0.299 \pm 0.006$ & $0.245 \pm 0.012$ & 2.3 \\
NGC 4418 & $\mathrm{HCO}^{+}(4-3)$ & $0.204 \pm 0.006$ & $0.343 \pm 0.028$ & 0.51 \\
Titan & 0.85 & $3.41 \pm 0.08$ & $0.848 \pm 0.008$ & 1.9 \\
Titan & 0.88 & $3.28 \pm 0.06$ & $0.863 \pm 0.007$ & 1.9 \\
J1222+042 & 0.85 & $0.55 \pm 0.02$ & $0.049 \pm 0.023$ & 2.8 \\
J1222+042 & 0.88 & $0.53 \pm 0.01$ & $0.0 \pm 0.0$ & 3.2 \\
\hline
\end{tabular}

Notes. Fitting of NGC 4418 used data from multiple array configurations; 2010 VEX, SC, and 2012 EXT. The VEX data of $\mathrm{HCO}^{+}$had too low $\mathrm{S} / \mathrm{N}$ to be usable. Fitting of Titan and the quasar J1222+042 were to verify our data analysis. The size of Titan based on ephemeris is 0.'8425. Column 2: continuum wavelength or line name. The $0.85 \mathrm{~mm}$ continuum is from USB and $0.88 \mathrm{~mm}$ is from LSB. Column 3: flux density at zero baseline (i.e., total flux density). For lines, this is for the line emission averaged from 1900 to $2300 \mathrm{~km} \mathrm{~s}^{-1}$. Column 4: the size is FWHM of a Gaussian for quasars and NGC 4418, and the diameter of a uniform-brightness disk for Titan.

The dust opacity about 1 is favored, as was concluded by Lisenfeld et al. (2000), because most infrared luminous galaxies have $\beta \approx 1.5-2$ (Lisenfeld et al. 2000; Dunne \& Eales 2001). The observed $\alpha$ corresponds to $\tau=2.2 \pm 0.5$ and $1.8 \pm 0.5$ for $\beta=2$ and 1.5 , respectively. For the second model, we note that the SED slope does not become 2 at shorter wavelengths as expected for $\tau \gg 1$. The shallower slope of the Planck function compared to the R-J formula must partly be the reason. Another likely reason is that the dust distribution is not just a $0{ }^{\prime} .1$ slab of uniform opacity but presumably has a low opacity halo as suggested by the larger extent of line emission than the $860 \mu \mathrm{m}$ continuum. The spectral slope in the general multi-component case without mutual shielding is the flux-weighted mean of the spectral indexes, $\alpha=\sum S_{i} \alpha_{i} / \sum S_{i}$, where the $i$ th component has the flux density $S_{i}$ and spectral index $\alpha_{i}$. For $\alpha_{1}=2$ and $\alpha_{2}=4$, the observed $\alpha$ corresponds to a fraction of the optically thick core of $73 \% \pm 9 \%$. Thus, as long as we assume $\beta \approx 2$, we need an optically thick core $(\tau \gtrsim 1)$ that dominates the SED at $\lambda \sim 1 \mathrm{~mm}$. In addition to the two models, the high opacity is also supported by the high brightness temperature that sets a lower limit of about 0.1 to the $860 \mu \mathrm{m}$ opacity from the constraint that opacity-corrected brightness temperature cannot exceed dust sublimation temperature.
There are other factors that depend on frequency and potentially affect the spectral index. They include the core size defined with the $\tau=1$ surface, the dust temperature at the photosphere, and absorption of the core emission by dust around it. The last one is addressed in Section 3.3.4.

\subsubsection{Gas Column Density, Mean Density, and Mass}

The dust continuum opacity $\tau_{860 \mu \mathrm{m}} \approx 1$ of the core translates to a hydrogen column density $N_{\mathrm{H}} \sim 10^{25.7} \mathrm{~cm}^{-2}$ for the dust opacity to column density relation $N\left(\mathrm{H}+\mathrm{H}_{2}\right) / \tau_{\lambda}=1.2 \times$ $10^{25}(\lambda / 400 \mu \mathrm{m})^{2} \mathrm{H}$-atom $\mathrm{cm}^{-2}$ (Keene et al. 1982; Hildebrand 1983). Since the core has a size of $\sim 20$ pc (0.' 1 in FWHM from Section 3.2.4; see the next subsection for two models), we obtain $n_{\mathrm{H}_{2}} \sim 5 \times 10^{5} \mathrm{~cm}^{-3}$ for the mean gas density (i.e., number density of hydrogen molecules $)$ and $M_{\text {mol }}(r \leqslant 10 \mathrm{pc}) \sim$ $1 \times 10^{8} M_{\odot}$ for the total gas mass in the core assuming that the gas is mostly molecular there. The total mass depends on the density distribution. The estimate above is for a uniform density and is an upper limit if the gas density increases toward the center.

\subsubsection{Brightness Temperature and Luminosity}

Table 6 lists the deconvolved (peak) brightness temperature and the bolometric luminosity of the nucleus. The luminosity is derived from the $860 \mu \mathrm{m}$ core size and the temperature. Here we use two different models to estimate the brightness temperature and the bolometric luminosity of the nucleus as we did in S08 for Arp 220. One is the circular Gaussian we used for the visibility fit above and the other is a circular disk with uniform brightness. The Gaussian model describes dust that is not fully opaque across the nucleus and that may also have a radial temperature gradient. The circular disk model corresponds to a sphere of uniform surface brightness projected on the sky. Both models can fit the same data well when the visibilities are sampled only within the half-maximum baseline length of the visibility function. The diameter of the model disk is 1.6 times the FWHM of the Gaussian in such a case (S08). For the luminosity calculation, it is assumed that the $860 \mu \mathrm{m}$ emission is optically thick in the uniform disk/sphere model. Since the deconvolved $T_{\mathrm{b}}$ was used without any opacity correction (i.e., not divided by $\left.1-e^{-\tau}\right)$, the derived temperature and luminosity are lower limits. We calculated the luminosity of the Gaussian model by using its FWHM size and its peak brightness temperature. This crudely approximates the effect mentioned earlier that the size of the photosphere should increase toward higher frequencies as dust opacity increases with frequency.

Table 6

Luminosity Estimates of the Nucleus

\begin{tabular}{lcccccc}
\hline \hline Shape & \multicolumn{2}{c}{ Size } & $T_{\mathrm{b}}$ & $\log L_{\text {bol }}$ & \multicolumn{1}{l}{$\log \Sigma\left(L_{\text {bol }}\right)$} & \multicolumn{1}{l}{$\log \rho\left(L_{\text {bol }}\right)$} \\
\cline { 2 - 7 } & $(\mathrm{mas})$ & $(\mathrm{pc})$ & $(\mathrm{K})$ & $\left(L_{\odot}\right)$ & $\left(L_{\odot} \mathrm{pc}^{-2}\right)$ & $\left(L_{\odot} \mathrm{pc}^{-3}\right)$ \\
$(1)$ & $(2)$ & $(3)$ & $(4)$ & $(5)$ & $(6)$ & $(7)$ \\
\hline Gaussian & $101 \pm 11$ & $17 \pm 2$ & $205 \pm 47$ & $11.3 \pm 0.3$ & $9.0 \pm 0.4$ & $8.0 \pm 0.4$ \\
Disk/sphere & $162 \pm 18$ & $27 \pm 3$ & $119 \pm 27$ & $10.8 \pm 0.3$ & $8.1 \pm 0.4$ & $6.8 \pm 0.4$ \\
\hline
\end{tabular}

Notes. Column 1: assumed shape of the source to calculate the peak brightness temperature. Disk is a circular disk (i.e., a sphere projected on the sky) with uniform surface brightness. Columns 2 and 3: source size is the FWHM for the Gaussian model and the diameter for the disk/sphere model. Column 4: brightness temperature calculated with the Planck function. This is the peak value for the Gaussian model and the value across the surface in the disk/sphere model. Column 5: bolometric luminosity of the source calculated from the source size, temperature, and the Stefan-Boltzmann law; see Section 3.3.3. Columns 6 and 7: the mean surface density and volume density of the luminosity within the size in Column 3. 
The derived luminosity of the nucleus is about $1 \times$ $10^{11} L_{\odot}$, which is comparable to the total luminosity of the galaxy $\left(10^{11.1} L_{\odot}\right.$, Table 1$)$. We obtained nuclear luminosities $\log \left(L_{\mathrm{bol}} / L_{\odot}\right)=11.3 \pm 0.3$ and $10.8 \pm 0.3$ for the two models. Luminosity surface density and volume density are also calculated for each model and listed in Table 6 for the central $\sim 20 \mathrm{pc}$. The errors here and in Table 6 are random errors due to the $5 \%$ flux error and the $11 \%$ size uncertainty and do not include systematic error due to the models.

There are two notable sources of systematic errors in the derived parameters in addition to the assumptions on the source shape and on the $860 \mu \mathrm{m}$ opacity. (The underestimating effect of the latter is minor as noted in S08.) One is the absorption of the core emission by the surrounding dust and gas. It arises because the $\sim 0^{\prime} .1$ ( $\sim 20 \mathrm{pc}$ ) continuum core is embedded in an order of magnitude larger envelope as we saw in Section 3.2. The continuum emission from the core must suffer from extinction to some extent by the envelope through continuum self-absorption and line absorption by various molecules. Our continuum brightness temperature derived without the extinction correction is therefore a lower limit of the true brightness temperature at the surface of the $\sim 0^{\prime \prime} .1$ core. The correction for it would increase the core luminosity. We will analyze this submillimeter extinction a little more in Section 3.3.4 and find the extinction to be about 5\% at $860 \mu \mathrm{m}$. The other source of systematic error is the possible contamination of the $860 \mu \mathrm{m}$ continuum by molecular lines. This effect can be estimated to be about $10 \%$ assuming that the spectral index between lower and upper sidebands obtained in the SC configuration, $6.25 \pm 0.84$ (Section 3.2.3), is due to line contamination to the USB data and that the true continuum spectral index is $2.55 \pm 0.18$ (Section 3.3.1). The continuum brightness temperature of the core would be lower without the contamination. However, this does not necessarily mean that the bolometric luminosity of the core is overestimated by this effect. Thermal lines can significantly add flux density to the $860 \mu \mathrm{m}$ continuum only when the line and continuum opacities satisfy $\tau_{\text {line }}>\tau_{\text {cont }}$ and $\tau_{\text {cont }} \lesssim 1$, because no thermal line will be seen from a blackbody. Since we use the brightness temperature of the continuum core for our luminosity estimate assuming that the continuum is optically thick, the line emission, if it is from the 0.1 core itself, would (partly) compensate for the decrement of the observed continuum brightness temperature due to $\tau_{\text {cont }}$ that is not $\gg 1$.

In addition to these two error sources, our visibility fits are insensitive to a point-like source with a small flux contribution. This is due to our $u-v$ coverage limit. For example, a $20 \mathrm{mJy}$ source with a size of 20 mas ( 3 pc) can be undetected in our visibility fit (Figure 9(a)) and would have a temperature of $700 \mathrm{~K}$ and luminosity of $10^{12} L_{\odot}$. The total luminosity of the galaxy limits the presence of such compact sources.

\subsection{4. $450 \mu \mathrm{m}$ Continuum}

We detected $450 \mu \mathrm{m}$ continuum at $5.8 \sigma$ with the resolution of $0.23 \times 00^{\prime \prime} .15$ at the position of the $860 \mu \mathrm{m}$ continuum peak (Figure $8(1)$ ). The $450 \mu \mathrm{m}$ nucleus in the map appears unresolved but this is largely due to the low $\mathrm{S} / \mathrm{N}$. (Imagedomain fitting gives a nominal deconvolved size of 0!. $10 \pm 0$ '.04 in the major axis while the source is unresolved in the minor axis.) The $450 \mu \mathrm{m}$ detection of the subarcsec-scale nucleus is at $10 \sigma$ and hence even more secure when the data are convolved to 0.5 resolution (Table 4 ). (The $\mathrm{S} / \mathrm{N}$ increases because the convolution lowers the weight of noisy long baselines.) The peak and total flux densities in both resolutions are about $370 \mathrm{mJy}$ and $\mathrm{mJy}$ beam $^{-1}$, respectively.

The $450 \mu \mathrm{m}$ continuum flux density that we detected is significantly lower than that of the central $3 \mathrm{kpc}$ of the galaxy (see Figure 10). The ratio is $0.31 \pm 0.15$ between the SMA and single-dish measurements. The latter was made by Roche \& Chandler (1993) with the UKT14 bolometer, the filter of which had the effective frequency of $682 \mathrm{GHz}(440 \mu \mathrm{m})$ and the bandwidth of $84 \mathrm{GHz}$ (Duncan et al. 1990). We subtracted from the bolometer measurement the contribution of $\mathrm{CO}(6-5)$ line $(\sim 0.1 \mathrm{Jy}$, calculated from the $\mathrm{CO}(3-2)$ single-dish flux of Yao et al. (2003) scaled by a factor of four) and rescaled the continuum to our observing frequency using the spectral index $\alpha=2.55$. The single-dish flux density of the $450 \mu \mathrm{m}$ continuum estimated this way is $1.18 \pm 0.33 \mathrm{Jy}$. The low flux recovery ratio at $450 \mu \mathrm{m}$ suggests that there is a significant $450 \mu \mathrm{m}$ emission that is too extended for detection at our sensitivity and with the shortest baseline of $240 \mathrm{k} \lambda$ in the VEX configuration. We simulated the degree of flux recovery with our actual $u-v$ coverage for Gaussian sources of various sizes. (We did not fit the visibilities unlike our $860 \mu \mathrm{m}$ data analysis because the $450 \mu \mathrm{m}$ data have lower S/N.) Figure 11 shows that the size of the $450 \mu \mathrm{m}$ continuum source would be $0.24 \pm 0.07$ (FWHM) if the source were approximately a single Gaussian. The peak brightness temperature of the source would be $70 \pm 20 \mathrm{~K}$ and the bolometric luminosity of this source would be on the order of only $10^{10.2} L_{\odot}$, which disagrees with our estimate at $860 \mu \mathrm{m}$.

A two-component model with a core and an envelope agrees with the observations within the errors and is more reasonable in our view. The two components are introduced in view of the compact continuum core and the more extended $\mathrm{CO}$ emission both observed at $860 \mu \mathrm{m}$. They are treated separately to simplify our modeling though they are probably not two discrete entities. (More detailed multi-component models have been made by González-Alfonso et al. (2012b) and C12. Our intention here is not to update their models but to apply a simple model to understand our submillimeter continuum observations.) The core and envelope are assumed to have sizes of 0.11 and 0.5 (both in FWHM), respectively. Also the core is assumed to dominate the $860 \mu \mathrm{m}$ emission with $85 \%$ of the total flux density. The extended envelope with the small remaining flux density has too low a surface brightness to be detected in our high-resolution map. However, the amount of extended $860 \mu \mathrm{m}$ continuum in this model is implied by our $\mathrm{CO}(3-2)$ data. Namely, if one-third of the $\mathrm{CO}(3-2)$ emission is from the envelope region, then its flux of about $300 \mathrm{Jy} \mathrm{km} \mathrm{s}^{-1}$ and the typical $\mathrm{CO}(3-2)$ equivalent width of $1 \times 10^{4} \mathrm{~km} \mathrm{~s}^{-1}$ among infrared bright galaxies (Seaquist et al. 2004) suggest a $860 \mu \mathrm{m}$ extended continuum of $\sim 30 \mathrm{mJy}$, which is about $15 \%$ of the total continuum flux density. The analysis of spectral index in Section 3.3.1 also implied a small contribution to $860 \mu \mathrm{m}$ emission from an optically thin and extended component. We assume the spectral index between $860 \mu \mathrm{m}$ and $450 \mu \mathrm{m}$ to be 2 for the optically thick core and 4 for the optically thin envelope (i.e., the model assumes $\beta=2$ ). In addition, since the core is embedded in the envelope, the former should be extinguished by the latter. The opacity of the envelope to cover the core is assumed to be $\sim 1$ at $200 \mu \mathrm{m}$, which we took from the model of González-Alfonso et al. (2012b) noting that it makes the envelope optically thin at $860 \mu \mathrm{m}(\tau=0.05)$ as we assumed above. At $450 \mu \mathrm{m}$ the core is extinguished by the envelope with $\tau=0.2$ and the envelope itself is resolved out in our VEX data. Overall, this model predicts our flux recovery 

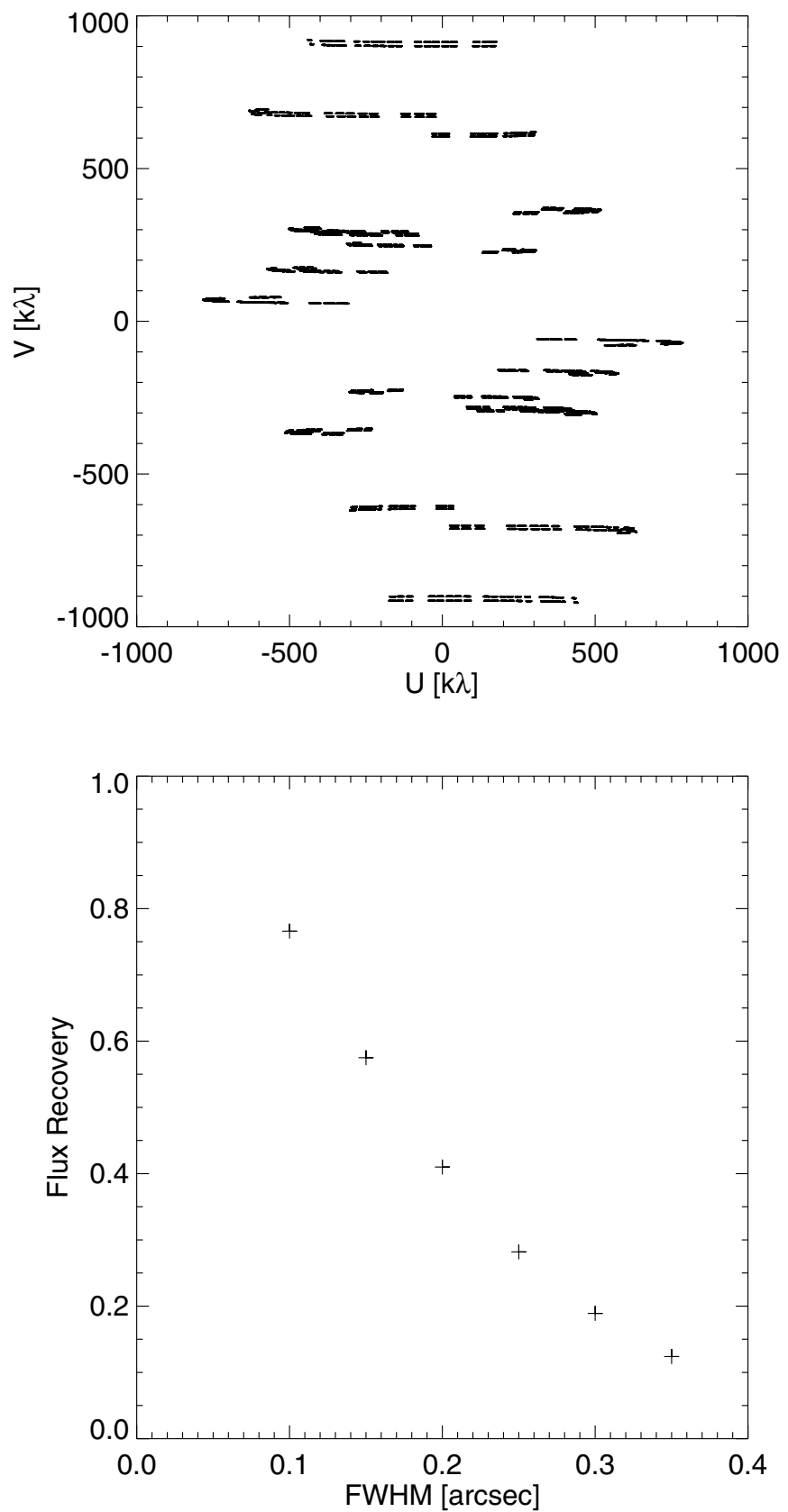

Figure 11. Top: sampling of our $660 \mathrm{GHz}$ observations in the $u-v$ plane. Bottom: fraction of flux recovered in the simulations of our observations for Gaussian sources with various sizes.

rate to be about $45 \%$, which agrees with our observed value of $31 \% \pm 15 \%$. The current data quality does not justify further parameter tuning and model refinement.

This model illustrates that two effects likely contribute to the lower flux recovery rate at $450 \mu \mathrm{m}$ than $860 \mu \mathrm{m}$. One is that the core emission is saturated while emission from the extended outer region is not. The other is that the emission from the hot nucleus is extinguished more at shorter wavelengths by the colder circumnuclear dust. These effects can also explain the $60 \mu \mathrm{m}$ peak of the SED of NGC 4418 despite the energetically dominant core emitting at $>100 \mathrm{~K}$ at $860 \mu \mathrm{m}$. The same effects probably explain similar observations in Arp 220 where the two nuclei contribute much less to the total at $435 \mu \mathrm{m}$ than at $860 \mu \mathrm{m}$ (Matsushita et al. 2009).
Table 7

Line Velocities and Widths

\begin{tabular}{lcc}
\hline \hline Line & $\begin{array}{c}V_{\mathrm{c}} \\
\left(\mathrm{km} \mathrm{s}^{-1}\right)\end{array}$ & $\begin{array}{c}\text { FWHM } \\
\left(\mathrm{km} \mathrm{s}^{-1}\right)\end{array}$ \\
\hline $\mathrm{HCO}^{+}(4-3)$ & $2088.3 \pm 4.2$ & $235.1 \pm 9.8$ \\
$\mathrm{HCN}(4-3)$ & $2085.8 \pm 2.8$ & $252.7 \pm 6.6$ \\
$\mathrm{CO}(3-2)$ & $2097.8 \pm 0.2$ & $142.2 \pm 0.5$ \\
$\mathrm{CS}(7-6)$ & $2088.7 \pm 4.6$ & $225.8 \pm 10.8$ \\
$\mathrm{~N}_{2} \mathrm{H}^{+}(3-2)$ & $2099.8 \pm 5.4$ & $143.8 \pm 12.8$ \\
$\mathrm{HCO}^{+}(3-2)$ & $2088.9 \pm 3.5$ & $197.9 \pm 8.2$ \\
$\mathrm{HCN}^{(3-2)}$ & $2089.9 \pm 3.0$ & $244.9 \pm 7.9$ \\
$\mathrm{CO}(2-1)$ & $2100.4 \pm 0.3$ & $142.4 \pm 0.7$ \\
\hline
\end{tabular}

Notes. Centroid velocities and widths of molecular lines in the central 6 " of NGC 4418. The velocities are in the radio definition and with respect to the LSR. These parameters are from Gaussian fitting to the spectra in S10. Errors are $\pm 1 \sigma . \mathrm{HCN}(4-3)$ is blended with a $\mathrm{HC}_{3} \mathrm{~N}$ line on the low-velocity side.

\subsection{Kinematics of Molecular Gas}

The HCN velocity map (Figure 8(f)) shows in the central $0.5(80 \mathrm{pc})$ a systematic gradient of mean velocity in the northeast-southwest direction, which is close to the galaxy major axis (P.A. $=60^{\circ}$, Table 1 ). The HCN channel maps in Figure 6 show this velocity structure as the shift of the emission peak across the continuum position from southwest (lower right) to northeast (upper left) in channels between 1990 and $2200 \mathrm{~km} \mathrm{~s}^{-1}$. In Figure 12, moment maps of $\mathrm{HCN}, \mathrm{HCO}^{+}$, and CS confirm the presence of the gradient across the nucleus although the direction of the velocity gradient is closer to the north-south direction in these lower resolution data. This is also the case in the $\mathrm{CO}$ high-resolution data (Figure 8(b)) in the central 0.5 .

We attribute the central velocity gradient to rotation around the luminous core and estimate its dynamical mass to be $M_{\text {dyn }}(r \leqslant 15 \mathrm{pc}) \sim 2 \times 10^{8} M_{\odot}$. We estimated for this a velocity shift of about $400 \mathrm{~km} \mathrm{~s}^{-1}$ in $0^{\prime \prime} .2$ across the nucleus from the CO and $\mathrm{HCN}$ position-velocity (PV) diagrams in Figure 13, where almost the full velocity range of each line is observed at the central $\sim 00^{\prime} 3$. The Keplerian dynamical mass above is for the $62^{\circ}$ inclination of the galaxy. Because of the insufficient spatial resolution to obtain an accurate rotation curve, our $M_{\text {dyn }}$ estimate is admittedly crude and its error may be as large as a factor of two even if the adopted inclination is correct. If the rotation velocity increases toward the center at these scales, as we will see possible below, the dynamical mass is more likely to be overestimated. For comparison, we estimated in our companion paper the mass of the central black hole in this galaxy to be $9 \times 10^{6} M_{\odot}(\mathrm{C} 12$, for the distance of $34 \mathrm{Mpc})$. This value follows from the $K$-band bulge luminosity $10^{9.8} L_{\odot, K}$, which is calculated from the total galaxy luminosity in Table 1 and the mean bulge-to-total luminosity ratio of 0.31 for Sa galaxies (Graham \& Worley 2008). The mass should have a factor of two uncertainty due to the scatter in the $L_{K}$,bulge $-M_{\mathrm{bh}}$ correlation (Marconi \& Hunt 2003).

We also infer from information of multiple lines that gas motion is faster toward the center. Figure 14 and Table 7 show the centroid velocities and widths of the molecular lines measured in the SC configuration data of S10. An interesting observation here is that there are two groups in the centroid velocity-line FWHM plane. The first group consists of $\mathrm{CO}(3-2), \mathrm{CO}(2-1)$, and $\mathrm{N}_{2} \mathrm{H}^{+}(3-2)$ and have centroid velocities $V_{\mathrm{c}} \approx 2100 \mathrm{~km} \mathrm{~s}^{-1}$ and FWHM $\approx 142 \mathrm{~km} \mathrm{~s}^{-1}$. The rest of the lines form the second group at $V_{\mathrm{c}} \approx 2088 \mathrm{~km} \mathrm{~s}^{-1}$ and FWHM $\approx 234 \mathrm{~km} \mathrm{~s}^{-1}$. 

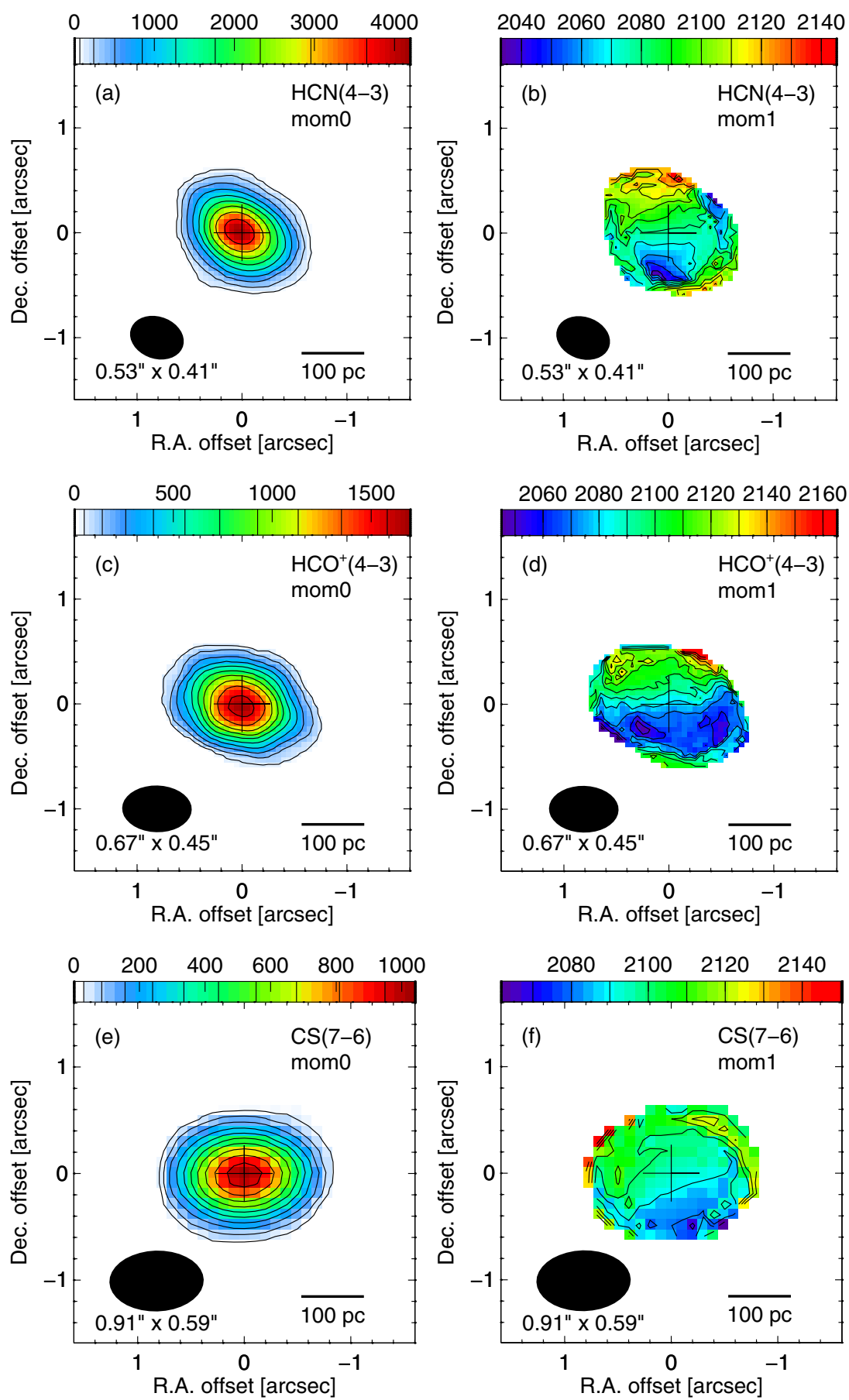

Figure 12. Integrated intensity and mean velocity maps of $\mathrm{HCN}(4-3), \mathrm{HCO}^{+}(4-3)$, and $\mathrm{CS}(7-6)$ emission. Data from three array configurations are used except for CS that was observed in two. The $n$th contour is at $70 n^{1.8}, 50 n^{1.5}$, and $30 n^{1.5} \mathrm{~K} \mathrm{~km} \mathrm{~s}^{-1}$ in (a), (c), and (e), respectively. Velocity contours are in $10 \mathrm{~km} \mathrm{~s} \mathrm{~s}^{-1}$ steps. The position of the $860 \mu \mathrm{m}$ continuum peak, whose coordinates are in Table 1, is marked with the plus sign in each panel and is used as the origin of the offset coordinates. The black ellipses show the FWHM sizes of the synthesized beams.

(A color version of this figure is available in the online journal.)

Also, there is a trend that lines with higher critical densities are wider. This variation of line parameters again suggests that the molecular gas in the center of NGC 4418 has non-uniform properties or multiple components. Indeed, the latter trend can be easily explained if the gas motion around the nucleus is faster closer to the center and if denser gas is more localized around the nucleus. This model agrees with our previous observation in Table 4 that the lines with higher critical densities are more concentrated in the nucleus than $\mathrm{CO}$. If lines with higher critical densities indeed trace gas closer to the nucleus, and if we also assume that the gas distribution is axisymmetric around the nucleus, the true systemic velocity of the nucleus would be about $2088 \mathrm{~km} \mathrm{~s}^{-1}$.

We can also evaluate radial motion of molecular gas from our data. HCN spectra near the continuum peak in Figure 15 and the HCN PV diagram (Figure 13) show a minor depression $(2 \sigma)$ at $2080 \mathrm{~km} \mathrm{~s}^{-1}$. This may be due to gas at about $V_{\text {sys }}$ and through line self-absorption or line absorption of the continuum 

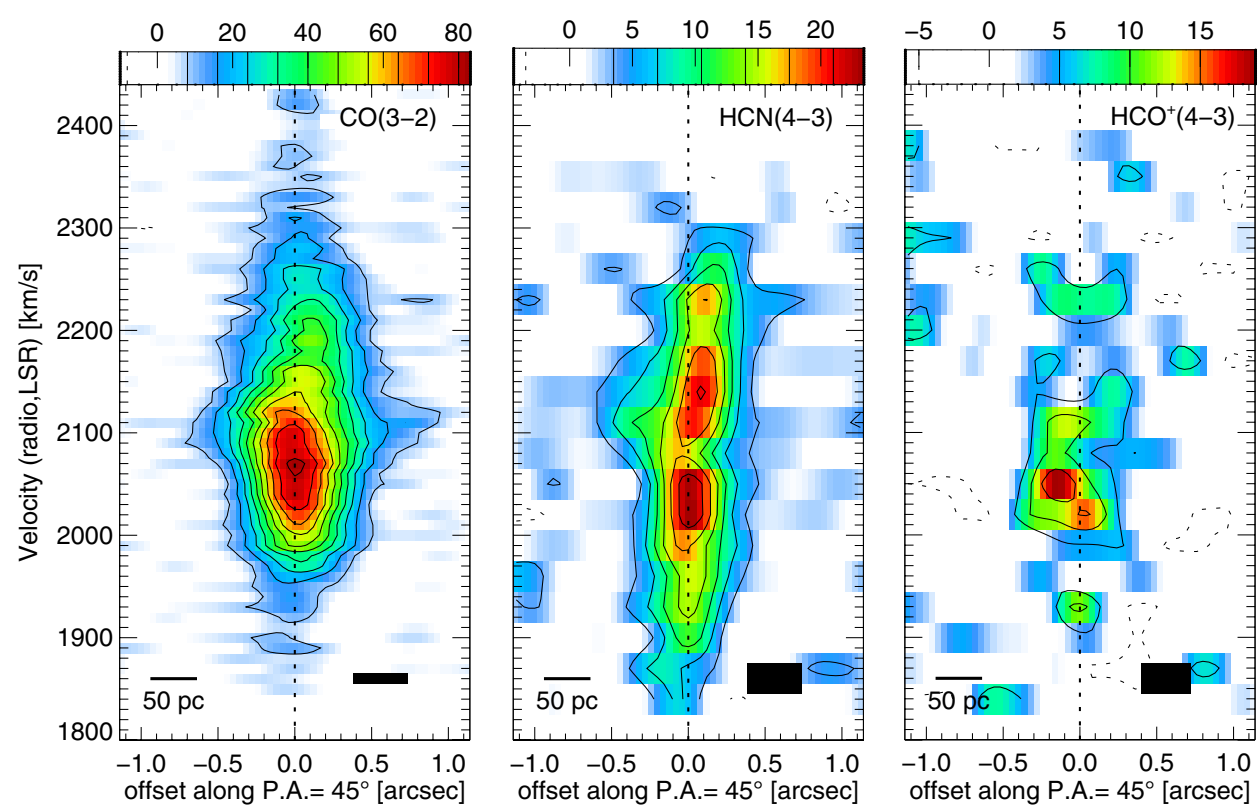

Figure 13. Position-velocity diagrams of molecular lines at the center of NGC 4418. Each PV cut is through the continuum peak and along the position angle of $45^{\circ}$ The origin of the abscissa is the continuum peak. The black rectangles at the bottom right show the spatial and velocity resolutions of the data. Contour steps are $8 \mathrm{~K}$ $(3.3 \sigma)$ for $\mathrm{CO}, 3.5 \mathrm{~K}(1.6 \sigma)$ for $\mathrm{HCN}$, and $5 \mathrm{~K}(1.6 \sigma)$ for $\mathrm{HCO}^{+}$. The highest velocity emission in the $\mathrm{CO}$ diagram is from $\mathrm{H}^{13} \mathrm{CN}$, and the low-velocity signal in the $\mathrm{HCN}$ diagram likely contains $\mathrm{HC}_{3} \mathrm{~N}(39-38)$.

(A color version of this figure is available in the online journal.)
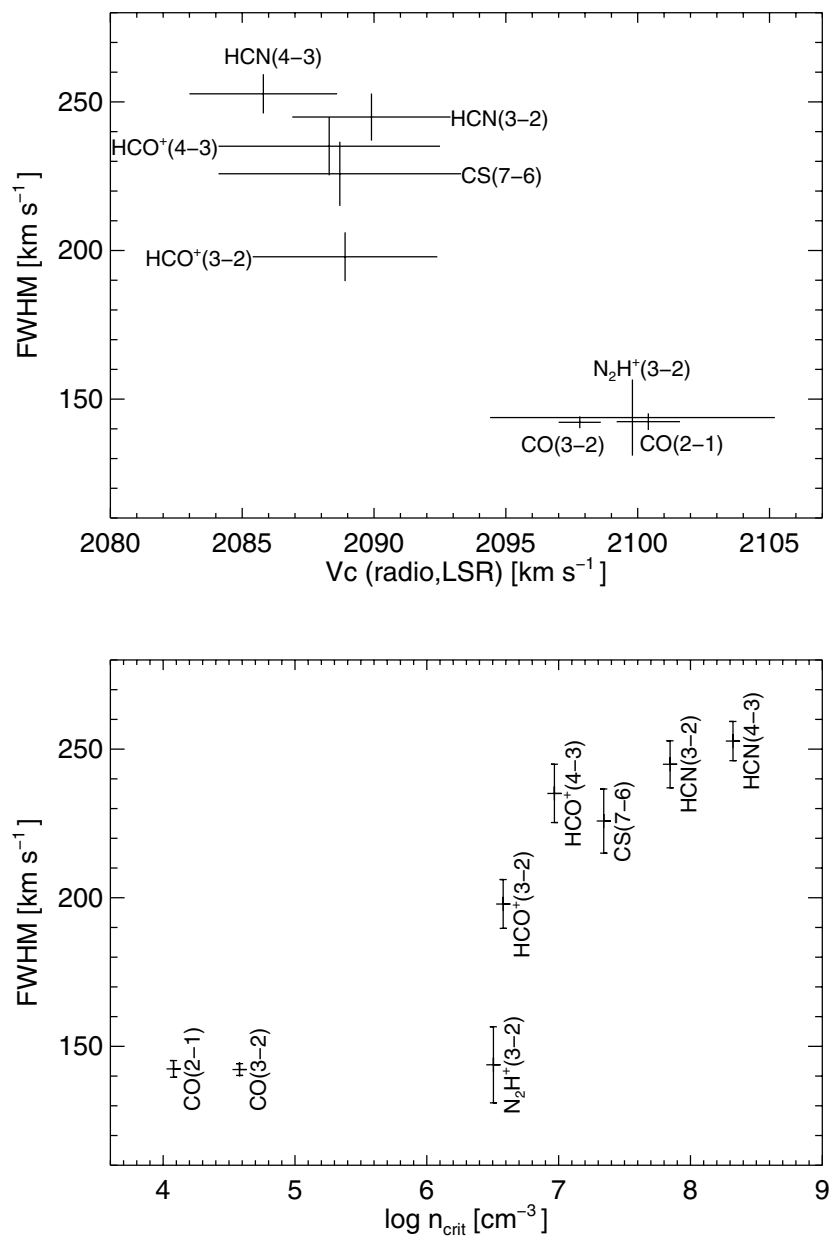

Figure 14. Top: line centroid velocity $\left(V_{\mathrm{c}}\right)$ and FWHM for the lines in Table 7. Bottom: critical density $\left(n_{\text {crit }}\right)$ at $50 \mathrm{~K}$ and FWHM for the same lines. Error bars are $\pm 4 \sigma$ for $\mathrm{CO}$ and $\pm 1 \sigma$ for others. Molecular parameters for the $n_{\text {crit }}$ are from the Leiden database (Schöier et al. 2005). from the core. The velocity of this possible absorption and the absence of clear absorption off the systemic velocity indicate little radial motion of the $\mathrm{HCN}$ gas. Meanwhile, the $\mathrm{CO}$ emission is significantly brighter below about $2100 \mathrm{~km} \mathrm{~s}^{-1}$ than at higher velocities in the PV diagram. See also the $\mathrm{CO}$ line profile at the continuum core position in Figure 15 where the line profile is asymmetric. If this is due to self-absorption of the $\mathrm{CO}$ emission, the foreground gas causing the absorption (and weaker $\mathrm{CO}$ emission) is redshifted with respect to the systemic velocity and hence is flowing inward. Although this asymmetry can be due to a chance imbalance of gas distribution between approaching and receding halves around the galactic center, this is qualitatively consistent with the redshifted $\mathrm{O}$ I, OH, and H I absorption observed by González-Alfonso et al. (2012b) and $\mathrm{C} 12$. The plausible gas motion mentioned here is opposite to that in Arp 220 where P-Cygni profiles of molecular lines suggested molecular outflows (Sakamoto et al. 2009).

Gas motion outside the central 0'.5 does not appear to be ordinary rotation in our data. The $\mathrm{CO}$ mean velocity maps in Figures 7(b) and 8(b) do not show an ordinary spider pattern of a rotating gas disk at $r \geqslant 0$.'25. The velocity maps probably contain a mixture of rotational and radial motions and appear complex because of non-uniform gas distribution and optical thickness of the $\mathrm{CO}$ line.

\subsection{Gas Properties}

Our high-resolution observations also provide the following new insights into gas properties in the nucleus.

First, the $\mathrm{CO}(3-2)$ line has a peak brightness temperature of $90 \mathrm{~K}$ at 0.3 resolution (Table 4 ), indicating warm molecular gas around the nucleus. The brightness temperature of thermalized $\mathrm{CO}$ is the gas kinetic temperature diluted by the $\mathrm{CO}$ opacity and by the observing beam if it is larger than the source. Both dilution effects are probably small for the extended ${ }^{12} \mathrm{CO}$ in this case. To put the observed $T_{\mathrm{b}}$ into context, the peak value of $90 \mathrm{~K}$ at $50 \mathrm{pc}$ resolution surpasses the peak $\mathrm{CO}$ brightness 

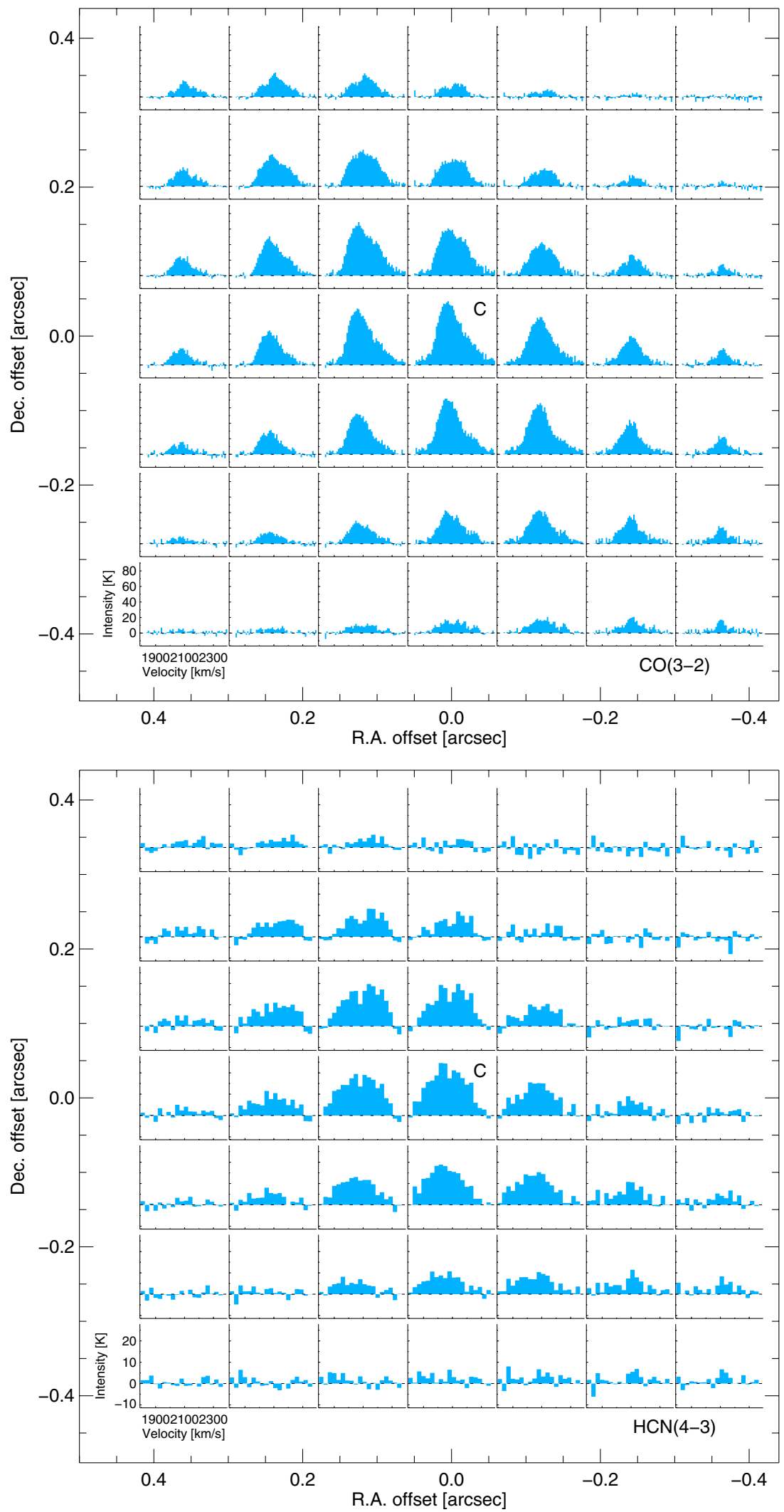

Figure 15. $\mathrm{CO}(3-2)$ and $\mathrm{HCN}(4-3)$ line profiles in the central arcsecond of NGC 4418, sampled on a grid of 0 '. 12 spacing. The origin of the offset coordinates is at the $860 \mu \mathrm{m}$ continuum peak, the spectrum observed at which is labeled with C. Velocity resolution of the spectra is 10 and $30 \mathrm{~km} \mathrm{~s}^{-1}$ for CO and $\mathrm{HCN}$, respectively. The rms noise per channel is 2.4 and $2.2 \mathrm{~K}$ for $\mathrm{CO}$ and $\mathrm{HCN}$, respectively.

(A color version of this figure is available in the online journal.) 
temperatures of about $50 \mathrm{~K}$ observed in Arp 220 and NGC 253 at $100 \mathrm{pc}\left(0^{\prime \prime} .3\right)$ and $20 \mathrm{pc}\left(1^{\prime \prime}\right)$ resolutions, respectively (Sakamoto et al. 2008, 2011). Meanwhile, both $\mathrm{HCN}(4-3)$ and $\mathrm{HCO}^{+}(4-3)$ have peak brightness temperatures of about $30 \mathrm{~K}$ at the same 0 .'3 resolution (Table 4). Their $\sim 3$ times lower $T_{\mathrm{b}}$ than that of $\mathrm{CO}$ must be largely due to beam dilution effects for these lines because they were found more compact than $\mathrm{CO}(3-2)$ in Section 3.2. Subthermal excitation and lower opacities of $\mathrm{HCN}$ and $\mathrm{HCO}^{+}$may be additional reasons for the lower $T_{\mathrm{b}}$ of these lines.

Second, the detection of lines from vibrationally excited molecules is another indication of high temperatures in the nucleus. The $\mathrm{HCN}$ and $\mathrm{HC}_{3} \mathrm{~N}$ lines that we detected from their vibrationally excited states have upper energy levels 510-1070 K above the ground level. Vibrational temperatures of these molecules have been estimated to be around $300 \mathrm{~K}$ (Costagliola \& Aalto 2010; S10; C12). Infrared radiation has been suggested to play a significant role for the vibrational excitation in the $\sim 100-200 \mathrm{~K}$ gas and dust that we inferred from brightness temperatures. Our companion paper (C12) presents a more detailed analysis and discussion on the excitation.

Third, the mean gas density $n_{\mathrm{H}_{2}} \sim 5 \times 10^{5} \mathrm{~cm}^{-3}$ of the $\sim 20$ pc core (Section 3.3.2) is high enough for $\mathrm{CO}$ excitation to $J=3$ but not for $\mathrm{HCN}$ and $\mathrm{HCO}^{+}$to $J=4$. Their critical densities for collisional excitation at $100 \mathrm{~K}$ are $\sim 10^{4.5}, 10^{7}$, and $10^{8} \mathrm{~cm}^{-3}$, respectively. The mean density is also far short of the $\sim 10^{11} \mathrm{~cm}^{-3}$ critical density to vibrationally excite $\mathrm{HCN}$ with $\mathrm{H}_{2}$ collisions. Therefore, the excitation of the lines with high critical densities are probably achieved through photon trapping, non-uniform density distribution to allow higher local densities than the mean, mid-infrared radiation, and/or electron collisions (S10; C12).

Fourth, the ratios between lines and between line and continuum vary with the area size of the measurements in the way expected when the ISM is warmer and denser at smaller radii. This is a consequence of the size variation among various emission lines (Section 3.2). For example, the $\mathrm{HCN}(4-3) / \mathrm{CO}(3-2)$ ratio of integrated brightness temperatures is $0.32 \pm 0.03$ and $0.170 \pm 0.004$ at 0.5 and $6^{\prime \prime}$ apertures, respectively. The ratio is expected to be higher when molecular gas is denser, warmer, or favorable for HCN line emission in other ways. Similarly, the equivalent widths of lines with respect to the continuum depend on the size scale. The $\mathrm{CO}(3-2)$ equivalent width changes by a factor of three, between $1.4 \times 10^{3}$ and $4.2 \times 10^{3} \mathrm{~km} \mathrm{~s}^{-1}$, from $0^{\prime \prime} .5$ to $6^{\prime \prime}$ scales. The smaller equivalent width at the smaller scale can be explained by the higher opacity of the dust continuum at the continuum core (S08). Finally, the ratio between $\mathrm{HCN}(4-3)$ and $\mathrm{HCO}^{+}(4-3)$ is $2.08 \pm 0.27$ in the central $0^{\prime \prime} 5$, while it is $1.65 \pm 0.07$ in the central $6^{\prime \prime}(\mathrm{S} 10)$. Among other differences, $\mathrm{HCN}$ has an order of magnitude higher critical density for collisional excitation and is also easier to radiatively excite than $\mathrm{HCO}^{+}(\mathrm{S} 10)$. See Section 4.1.8 for more discussion on this line ratio.

\subsection{Optical Color Index}

We found a U-shaped red feature in the multi-band optical images of the galaxy from the Sloan Digital Sky Survey (SDSS). Figure 16 shows the distribution of a flux ratio involving four SDSS bands, $\left(i^{\prime}+z^{\prime}\right) /\left(g^{\prime}+r^{\prime}\right)$. Areas brighter at longer wavelengths $\left(i^{\prime}\right.$ and $\left.z^{\prime}\right)$ are shown in red, while areas of lower flux ratios are in blue. The U-shaped red feature is along the northwestern semi-minor axis of the galaxy. No counterpart is visible around the southeastern semi-minor axis. The reddest

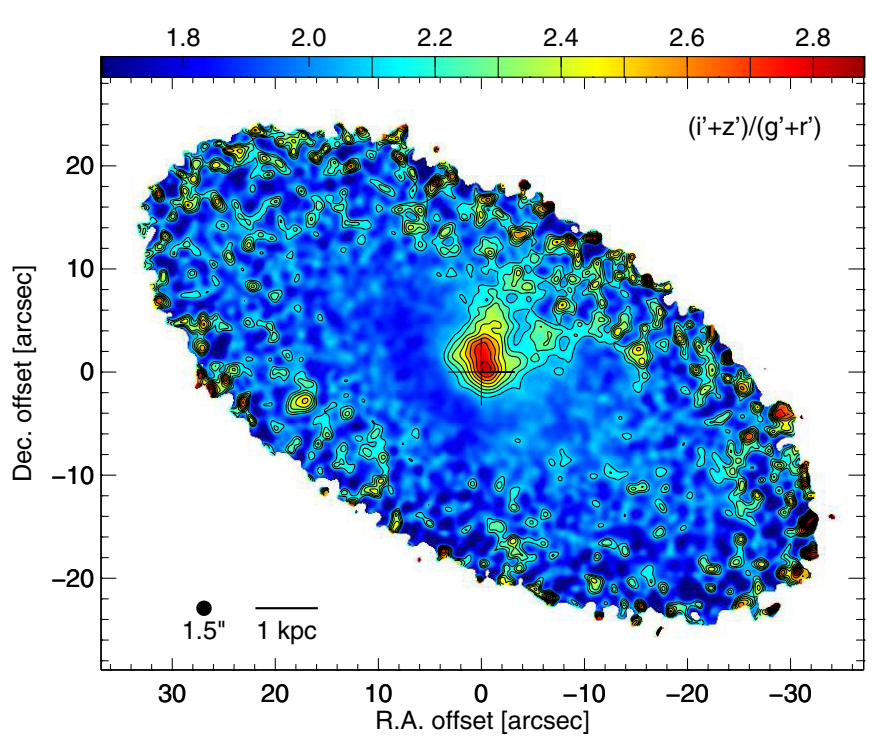

Figure 16. Distribution of optical color in the center of NGC 4418. Flux ratio involving four SDSS bands, $\left(i^{\prime}+z^{\prime}\right) /\left(g^{\prime}+r^{\prime}\right)$, is plotted in such a way that regions brighter in the longer wavelengths $i^{\prime}$ and $z^{\prime}$ than the shorter wavelengths $g^{\prime}$ and $r^{\prime}$ are shown in red, while low-ratio regions are shown in blue. The resolution of this image is $1^{\prime \prime} .5$. The origin of the offset coordinates is at the $860 \mu \mathrm{m}$ continuum peak and is marked with a plus sign. North is up and east is to the left.

point is almost on our $860 \mu$ m continuum peak (i.e., the nucleus) and is about $0.5 \mathrm{mag}$ (i.e., a factor $\sim 1.6$ ) redder than the offcenter region outside the $U$ shape. This feature extends at least up to $10^{\prime \prime}$ ( $1.7 \mathrm{kpc}$ on the sky) from the nucleus. The redder part of it near the nucleus has an elongation along the position angle of about $15^{\circ}$, similar to the faint $\mathrm{CO}(3-2)$ emission in Figure 7.

We interpret the red feature to be an outflow cone emanating from the galactic center along the rotation axis of the galaxy. The feature is very unlikely to be a structure on the galactic plane because such a feature would be torn by differential rotation and also because the feature is on the minor axis of the galaxy. The minor axis is determined by our viewpoint while a radial feature in the galactic plane should have no preferred position angle with respect to us. The outflow cone model does not have this problem because any feature along the rotation axis of the galaxy is always projected onto the minor axis. Also, the outflow can be bipolar in this model even though we only see half of it in the color index map. The fact that we see the red feature only on the northwestern side suggests the side to be the near side of the outflow and the far side of the galaxy disk, against which the outflow cone is visible as a red silhouette due to color-dependent extinction. Dust in the cone probably causes the extinction and the reddening. Our $\mathrm{CO}$ velocity data in Figure 7 cover only a small fraction of the red optical feature and do not reveal the gas motion in the red cone. The data only tell that the velocity field within a radius of $\sim 500 \mathrm{pc}$ is more complicated than expected from purely circular rotation around the center. However, Lehnert \& Heckman (1995) reported a $200 \mathrm{~km} \mathrm{~s}^{-1}$ velocity shift along the minor axis from their [N II] line measurements for a survey of starburst winds. The velocity shift suggests radial motion of the ionized gas along the polar axis. Although we cannot yet confirm an outward radial motion because the direction of the [N II] velocity shift was not given, the velocity shift along the red cone better fits the outflow interpretation. For this radial motion to be an inflow, gas and dust need to be falling in the U-shaped cone toward the nucleus 
along the polar axis, which seems too much of a coincidence. Our outflow model predicts that the gas on the northwestern cone should be blueshifted on average with respect to the nucleus.

Much closer to the nucleus, Evans et al. (2003) found radial dark lanes out to about $3^{\prime \prime}$ from the nucleus in their near-infrared data. The lanes have an average position angle of about $15^{\circ}$ and are on both sides of the nucleus. The fainter lane to the north is in the same direction as the reddest part of the optical U-shaped feature as well as the $\mathrm{CO}$ elongation. There might therefore be a change of the outflow direction, first at P.A. $\sim 15^{\circ}$ in the innermost region $\left(r<3^{\prime \prime} \approx 500 \mathrm{pc}\right)$ and then along the minor axis of the galaxy (P.A. $\sim-30^{\circ}$ ) outward.

We estimate the gas mass in the outflow to be $\sim 4 \times 10^{7} \mathrm{M}_{\odot}$. This is from the reddening and assumes a bipolar structure of the outflow. The excess color index in $g^{\prime}-r^{\prime}$ is $0.8 \mathrm{mag}$ at the central peak, about $0.5 \mathrm{mag}$ in the reddest $1 \mathrm{kpc}^{2}$ near the base of the $U$ shape, and 1 mag $\mathrm{kpc}^{2}$ when integrated over $2 \times 2 \mathrm{kpc}^{2}$ encompassing the entire $U$ shape. Adopting the Milky Way extinction law of $A_{g^{\prime}}-A_{z^{\prime}}=0.71 A_{V}$ and $\Sigma\left(\mathrm{H}+\mathrm{H}_{2}\right) / A_{V}=2 \times 10^{21} \mathrm{H} \mathrm{cm}^{-2}=16 M_{\odot} \mathrm{pc}^{-2}$, we estimate the gas column density to be $N_{\mathrm{H}}=2 \times 10^{21} \mathrm{~cm}^{-2}$ at the peak, the mean mass surface density in the reddest $1 \mathrm{kpc}^{2}$ to be about $10 M_{\odot} \mathrm{pc}^{-2}$, and the gas mass in the one side of the outflow (i.e., the U shape) to be about $2 \times 10^{7} M_{\odot}$. The mass in the entire outflow is twice the last value on the assumption of a symmetric bipolar structure. This mass estimate assumes foreground-screen extinction. The $860 \mu \mathrm{m}$ dust core mixed with stars in the galactic center does not contribute much to the reddening and, appropriately, to the mass estimated here. Note that the reddening data (and hence the outflow mass estimate from it) is more sensitive than our $\mathrm{CO}$ data in which the $\mathrm{U}$-shaped cone is not detected. The cone would have a CO line brightness temperature on the order of only $0.1 \mathrm{~K}$ when the outflow gas is $100 \%$ molecular, its $\mathrm{CO}$-to- $\mathrm{H}_{2}$ conversion factor is $2 \times 10^{20} \mathrm{~cm}^{-2}\left(\mathrm{~K} \mathrm{~km} \mathrm{~s}^{-1}\right)^{-1}$, and the line has a velocity width of $30 \mathrm{~km} \mathrm{~s}^{-1}$ at each position. This is about $0.5 \sigma$ in our $00^{\prime \prime} 6$ resolution $\mathrm{CO}(3-2)$ data cube.

We further estimate the kinematical age of the outflow to be on the order of $10 v_{200}^{-1} \mathrm{Myr}$ and the gas outflow rate $\sim 4 v_{200} M_{\odot} \mathrm{yr}^{-1}$. The parameter $v_{200}$ is the outflow velocity normalized by $200 \mathrm{~km} \mathrm{~s}^{-1}$ and is about unity for the projected minor-axis velocity shear of $200 \mathrm{~km} \mathrm{~s}^{-1}$ measured in [N II]. The age (i.e., crossing time) of the outflow is the ratio between the de-projected size of the outflow cone, $2 \mathrm{kpc}$, and the outflow velocity. The mass outflow rate from the nucleus is from the gas mass above and the crossing time. We caution about the uncertainties in these parameters due to the unknown velocities of various outflow media, the poorly constrained extent of the flow, and the uncertain projection effect. For example, the ionized gas traced by [N $\mathrm{II}]$ and the dust causing the reddening may have different bulk velocities. Also the opening angle of the outflow and the possible bend of the outflow direction add uncertainty to our outflow velocity and to the parameters dependent on it. For example, if the outflow velocity is as large as $1000 \mathrm{~km} \mathrm{~s}^{-1}$ seen in some molecular outflows (e.g., Chung et al. 2011; Sakamoto 2012), the crossing time will be reduced to $2 \mathrm{Myr}$ and the outflow rate will be $\sim 20 M_{\odot} \mathrm{yr}^{-1}$. Another caution is that, with the velocity information currently at hand, we cannot tell whether the gas and dust blown out from the nucleus to $\gtrsim 1 \mathrm{kpc}$ above the galactic plane will leave the galaxy or fall back to it. In the latter case, the mass-loss rate of the galaxy is smaller than the outflow rate from the nucleus.

\section{DISCUSSION}

\subsection{The Luminosity Source at the Nucleus}

We have obtained several parameters to constrain the nature of the luminosity source such as the $L_{\mathrm{bol}}, M_{\mathrm{dyn}}$, size, and $N_{\mathrm{H}}$ of the luminous core, and $\Sigma\left(L_{\mathrm{bol}}\right) \sim 10^{8.5} L_{\odot} \mathrm{pc}^{-2}$ and $L_{\text {bol }} / M_{\text {dyn }} \sim 500 L_{\odot} M_{\odot}{ }^{-1}$ calculated from them. While we derived these from our $860 \mu \mathrm{m}$ observations alone, they qualitatively agree with the observations of Roche et al. (1986) and many others that most of the luminosity of the galaxy must come from a compact and deeply enshrouded nucleus. The parameter values are comparable to or higher than those in the ultraluminous infrared galaxy Arp 220, where S08 obtained $L_{\text {bol }} / M_{\text {dyn }} \gtrsim 400 L_{\odot} M_{\odot}{ }^{-1}$ and $\Sigma\left(L_{\text {bol }}\right) \gtrsim 10^{7.6} L_{\odot} \mathrm{pc}^{-2}$ in the central $80 \mathrm{pc}$ of the western nucleus.

\subsubsection{Constraint from $L / M$}

A young starburst can have a bolometric luminosity-to-mass ratio $(L / M)$ at or above $1000 L_{\odot} M_{\odot}^{-1}$ for 3-10 Myr depending on the initial mass function (IMF), while a bare AGN at the Eddington luminosity has $L / M_{\mathrm{bh}} \approx 10^{4.5} L_{\odot} M_{\odot}{ }^{-1}$. Figure 17 shows this for starbursts simulated using Starburst99 (Leitherer et al. 1999, 2010; Vázquez \& Leitherer 2005) and the IMF of Kroupa (2002). The duration of $L / M \gtrsim 1 \times 10^{3} L_{\odot} M_{\odot}{ }^{-1}$ is about $5 \mathrm{Myr}$ for the normal (i.e., untruncated) Kroupa IMF in both instantaneous and continuous starbursts. In a continuous starburst, the combination of $L / M \approx 10^{3} L_{\odot} M_{\odot}{ }^{-1}$ and $L \approx 10^{11} L_{\odot}$ is obtained with the star formation rates (SFRs) of $30-100 M_{\odot} \mathrm{yr}^{-1}$ at the ages of 3-1 Myr. The starburst can be older and SFR lower if the IMF is biased toward high masses compared to the standard one (e.g., age $\sim 10 \mathrm{Myr}$ and SFR $\sim 10 M_{\odot} \mathrm{yr}^{-1}$ for the IMF mass range of $1-100 M_{\odot}$ ). The mass used for $L / M$ in Figure 17 is that of newborn stars (or the black hole mass for the Eddington limit). The dynamical mass in our observed $L_{\mathrm{bol}} / M_{\text {dyn }}$ includes the gas covering the nucleus, the central black hole, and the old stellar population pre-existing the starburst or nuclear activity. The gas mass is not negligible since it was estimated to be about half of the dynamical mass (Section 3.3.2). Removing these from the denominator of the observed $L / M \sim 500 L_{\odot} M_{\odot}{ }^{-1}$ will increase the ratio to $\gtrsim 1000 L_{\odot} M_{\odot}{ }^{-1}$. Figure 17 therefore suggests that if a starburst is responsible for the core luminosity of NGC 4418 its $L / M$ is near the maximum that a starburst can have only when it is young, assuming the standard IMF. The $L / M$ data from our submillimeter observations therefore provide a new way to constrain the age in the starburst model.

Regarding whether a compact and massive star cluster mentioned above is able to form, it has been argued that the maximum $L / M$ for a cluster-forming gas cloud or disk is 500-1000 $L_{\odot} M_{\odot}{ }^{-1}$ (Scoville 2003; Thompson et al. 2005). This limit is because too much luminosity will blow out the star forming gas with radiation pressure on dust and halt the star formation. The observed $L / M$ is close to this limit but does not clearly exceed it. Murray (2009) predicted a mass-size relation for massive clusters taking the radiation pressure into account. The diameters of $10^{7.5}$ and $10^{8} M_{\odot}$ clusters, which can have $\sim 10^{11} L_{\odot}$ when young, are 5 and $10 \mathrm{pc}$, respectively, in his relation. Thus, at least these considerations do not reject the starburst model with the parameters we currently have.

If the energy source of the $10^{11} L_{\odot}$ core is an accreting black hole then its Eddington ratio must be $\log \left(L / L_{\text {Edd }}\right)=$ $-0.5 \pm 0.3$ assuming the black hole mass of $10^{7.0 \pm 0.3} M_{\odot}$ (hence $L_{\text {Edd }}=10^{11.5 \pm 0.3} L_{\odot}$ ) estimated from the bulge luminosity 

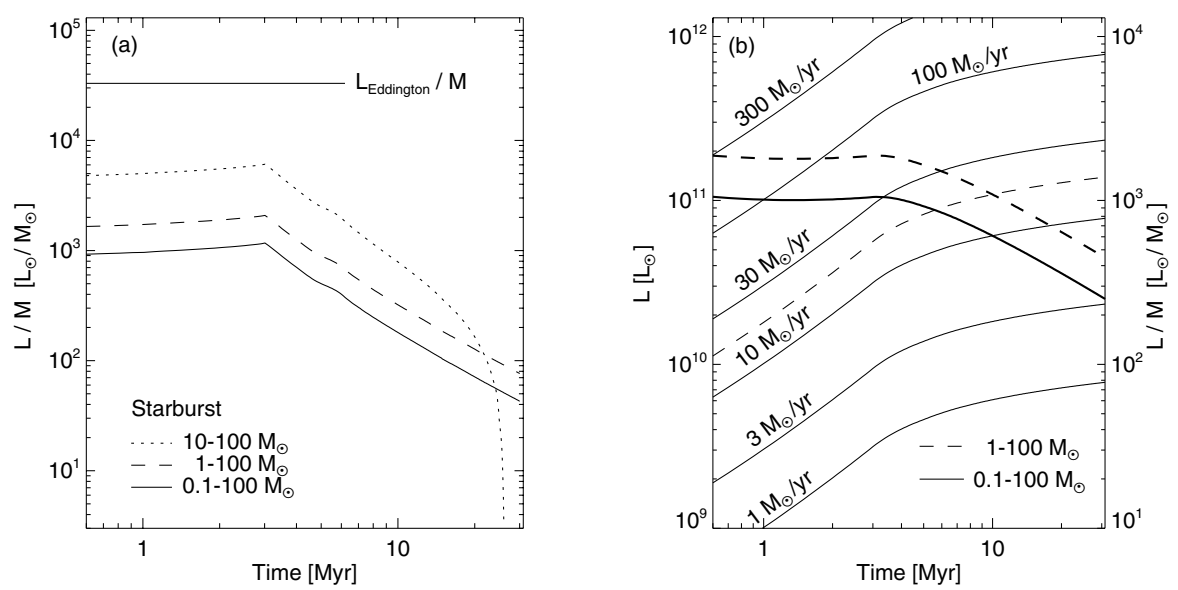

Figure 17. Evolution of starburst parameters simulated using Starburst99 and the IMF of Kroupa (2002), which has a power-law index of -1.3 for $0.1-0.5 M_{\odot}$ and -2.3 for $0.5-100 M_{\odot}$. (a) Luminosity-to-mass ratios for single-age (i.e., instantaneous) starburst populations with three different IMF mass ranges. Also plotted is the Eddington $L / M$ for fully ionized hydrogen plasma; the mass is the black hole mass in the context of AGN. (b) Luminosity and $L / M$ of continuous starbursts with different SFRs. Thin solid lines are starburst luminosities (left axis) for SFRs between 1 and $300 M_{\odot} \mathrm{yr}^{-1}$. The thin dashed line is the starburst luminosity for $\mathrm{SFR}=10 M_{\odot} \mathrm{yr}^{-1}$ and for the Kroupa IMF truncated to the range of $1-100 M_{\odot}$. The thick lines are the $L / M \mathrm{~s}$ (right axis); the dashed one is for the same truncated IMF as above. The $L / M$ ratio is independent of the SFR.

(C12; Section 3.4). Thus, an AGN has no problem to be the dominant energy source as long as the $L / M_{\text {bh }}$ is concerned. It has no problem to be smaller than the 20 pc core either.

\subsubsection{Constraint from $\Sigma\left(L_{b o l}\right)$}

The luminosity surface density that we obtained, $\Sigma\left(L_{\text {bol }}\right)=$ $10^{8.5 \pm 0.5} L_{\odot} \mathrm{pc}^{-2}$ in the central $20 \mathrm{pc}$ (Table 6), is among the highest compared to the mid-infrared survey of (ultra)luminous infrared galaxies, starbursts, and Seyfert nuclei by Soifer et al. (2000, 2001, 2003, 2004). In their 0.'3-0'.5 resolution survey, only 3 out of 22 nuclei were found to have a surface brightness (or its lower limit) at $10^{8} L_{\odot} \mathrm{pc}^{-2}$ or larger. All the three galaxies, Mrk 231, NGC 1275, and NGC 7469, have an AGN. Their mid-IR cores have (upper limit) sizes of about $20 \mathrm{pc}$ in two and $100 \mathrm{pc}$ in one. For comparison, the luminosity surface densities of star-formation-dominated nuclei are generally at or below $10^{7} L_{\odot} \mathrm{pc}^{-2}$. Between $10^{7}$ and $10^{8} L_{\odot} \mathrm{pc}^{-2}$ are ultraluminous infrared galaxies where both AGN and starburst may be hidden behind a large amount of gas and dust (Soifer et al. 2001). More recent observations by Imanishi et al. (2011) also support the mid-IR compactness and higher luminosity surface densities of AGNs compared to starbursts. There is a theoretical model explaining the apparent limit for starbursts around $10^{7} L_{\odot} \mathrm{pc}^{-2}$. In it a nuclear gas disk opaque at farIR will have that luminosity surface density when it supports itself by stellar radiation pressure and self-regulates to have Toomre's $Q \sim 1$ (Thompson et al. 2005). This is for dust temperatures less than about $200 \mathrm{~K}$ or for "warm starbursts" in Andrews \& Thompson (2011). For dust temperatures above $200 \mathrm{~K}$, which the $0^{\prime \prime} 1$ core of NGC 4418 may have, and for a gas surface density of $10^{5.8} M_{\odot} \mathrm{pc}^{-2}$ that corresponds to $\tau_{860 \mu \mathrm{m}} \sim 1$ of the continuum core, the disk at the Eddington limit is in the "hot starburst" regime and will have a surface brightness of $\sim 10^{8.5} L_{\odot} \mathrm{pc}^{-2}$ (Andrews \& Thompson 2011; see their Equation (7)). According to our data and this model, the nucleus of NGC 4418 has either an energetically dominant AGN or the hot starburst.

\subsubsection{Constraint from Mass Budget}

Our estimates of the masses in the central 30 pc of NGC 4418 are summarized in Table 8 . The mass of young stars there,
Table 8

Mass Budget in the Central $30 \mathrm{pc}$

\begin{tabular}{lcc}
\hline \hline Type & $\begin{array}{c}\log M \\
\left(M_{\odot}\right)\end{array}$ & Note \\
\hline$M_{\text {dyn }}(r \leqslant 15 \mathrm{pc})$ & $8.3 \pm 0.3$ & (a) \\
$M_{\text {bh }}$ & $7.0 \pm 0.3$ & $(\mathrm{a})$ \\
$M_{\text {mol }}(r \leqslant 10 \mathrm{pc})$ & $\sim 8.0$ & (b) \\
$M_{\text {young stars }}(r \leqslant 10 \mathrm{pc})$ & $8.0 \pm 0.5$ & (c) \\
$M_{\text {old stars }}(r \leqslant 15 \mathrm{pc})$ & $\sim 7.4$ & (d) \\
\hline
\end{tabular}

Notes. Numbers with $\sim$ may well have $0.3-0.5$ dex uncertainties as others.

a See Section 3.4.

b See Section 3.3.2.

c See Section 4.1.3. Most of the luminosity-bearing young stars must be well within the central 20 pc.

${ }^{\mathrm{d}}$ See Section 4.1.3. For an average Sa galaxy with $M_{K}=$ -23.8 mag. NGC 4418 is 0.3 times as luminous.

$10^{8.0 \pm 0.5} M_{\odot}$, is for a model young starburst having a luminosity of $10^{11} L_{\odot}$ and a plausible $L / M$ of $10^{3.0 \pm 0.5} L_{\odot} M_{\odot}{ }^{-1}$. The mean SFR of this starburst would be $10-100 M_{\odot} \mathrm{yr}^{-1}$ for a starburst age of $3 \mathrm{Myr}$. The mass of old stars in the central $30 \mathrm{pc}$, $\sim 10^{7.4} M_{\odot}$, is estimated for an average $\left(\sim L^{*}\right)$ Sa-type galaxy using the $K$-band photometric parameters of Graham \& Worley (2008, their Table 3), their conversion formulae to radial mass distribution (Terzić \& Graham 2005), and the $K$-band mass-tolight ratio of $1.06 M_{\odot} / L_{\odot K}$ for a $12 \mathrm{Gyr}$ old stellar population with $[\mathrm{Fe} / \mathrm{H}]=0$ (Worthey 1994). From the viewpoint of mass budget, AGN dominance in luminosity would eliminate the need for young stars and help fit the sum of the component masses to the dynamical mass. The information currently at hand, however, does not let us conclude the AGN dominance from the mass budget because the masses used here typically have an uncertainty of a factor of a few. The dynamical mass will be better constrained with higher resolution observations.

\subsubsection{Constraint from Timescale}

The current tentative estimate of the outflow kinematical age, $10 \mathrm{Myr}$, is marginally longer than the age that an energetically dominant starburst can have (Section 4.1.1). If this is firmly confirmed, it would be against the nascent starburst model. 


\subsubsection{Constraint from $N_{H}$}

The gas shroud around the nucleus can shield the X-rays from the putative AGN because it is highly Compton thick with $N_{\mathrm{H}} \gtrsim 10^{25} \mathrm{~cm}^{-2}$ to the galactic center (Section 3.3.2).

\subsubsection{Provisional Verdict}

To summarize on the luminosity source, the large luminosity of the nucleus can certainly be due to a hidden AGN as far as the parameters $L, L / M, \Sigma\left(L_{\mathrm{bol}}\right), N_{\mathrm{H}}$, and mass budget of the nucleus are concerned. It would have an Eddington ratio $\sim 0.3$ with a factor of two uncertainty if the black hole and the bulge follow the normal scaling relation. This bolometric Eddington ratio is high compared to the median ratio of $10^{-3}$ among local type 1 Seyferts (Ho 2008). In this sense the AGN mass accretion is rapid in NGC 4418 if the AGN is the dominant luminosity source. The black hole should be growing at a higher rate than in most low luminosity AGNs. The mass accretion rate to the black hole would be $0.1 M_{\odot} \mathrm{yr}^{-1}$ for a $10 \%$ radiative efficiency and the luminosity of $10^{11} L_{\odot}$ (i.e., $L_{\text {bol }} \approx 0.1 \dot{M} c^{2}$ ). The characteristic timescale for the black hole growth is $10^{8} \mathrm{yr}$ at this rate. This AGN gas consumption rate is much lower than the SFR required for the same luminosity because an AGN is more fuel-efficient.

A compact young starburst that has a mass $\sim 10^{8} M_{\odot}$, size $<20 \mathrm{pc}$, age $\sim$ a few Myr, and $L / M \sim 10^{3} L_{\odot} M_{\odot}{ }^{-1}$ is an alternative that has not been excluded with our observations. (The age could be up to $10 \mathrm{Myr}$ as far as $L / M$ is concerned, but starbursts older than $3 \mathrm{Myr}$ are less favored because supernova explosions would be destructive to the dusty core, as argued by Roussel et al. 2003.) While such a starburst would be at an edge of the parameter space allowed for starbursts, it is noteworthy that the massive concentration of dense molecular gas is a favorable environment for star formation. This is true even if there is a major AGN.

A combination of less luminous young starburst and a less luminous AGN is also possible as long as their total luminosity is about $10^{11} L_{\odot}$. It remains to be seen with more accurate mass, luminosity, and size measurements and the constraints mentioned above whether the nucleus of NGC 4418 is in the parameter region where only a dominant $\mathrm{AGN}$ is allowed.

\subsubsection{Consistency Check with Radio Data}

It is interesting to ask whether observations at radio wavelengths, which do not suffer from extinction by dust, are consistent with an energetically dominant starburst in the nucleus. As noted in Section 4.1.1, a starburst consistent with our SMA observations has an SFR of about $30 M_{\odot} \mathrm{yr}^{-1}$ in the last 3.3 Myr so that the starburst population has a stellar mass of $10^{8} M_{\odot}$, an $L / M$ of $10^{3} L_{\odot} M_{\odot}{ }^{-1}$, and the observed luminosity of $L_{\text {bol }} \sim 10^{11} L_{\odot}$. A younger starburst needs higher SFR to achieve the luminosity. We note that the $L_{\mathrm{IR}}-\mathrm{SFR}$ relation of Kennicutt (1998) and Kennicutt \& Evans (2012) gives $\mathrm{SFR} \approx 15 M_{\odot} \mathrm{yr}^{-1}$ for the $10^{11} L_{\odot}$. However, as one can see in Figure 17(b), a continuous starburst at that SFR will be about 10 Myr old when achieving the luminosity and by that time the $L / M$ will drop by about $40 \%$ from its peak to $\sim 600 L_{\odot} M_{\odot}{ }^{-1}$, assuming the standard Kroupa IMF. Thus, unless the IMF is truncated at low masses, our $L / M$ constraint prefers higher SFRs than estimated from the standard $L_{\mathrm{IR}}-\mathrm{SFR}$ relation.

We estimate a lower limit of SFR $\gtrsim 9 M_{\odot} \mathrm{yr}^{-1}$ using three constraints from the radio data and an SFR scaling relation of Murphy et al. (2011). The first constraint is the high brightness temperature of the nucleus, $\gtrsim 10^{5} \mathrm{~K}$ at $\sim 2 \mathrm{GHz}$ as reviewed in Section 1.1, at the compact nuclear source $\left(\sim 0^{\prime \prime} .1-00^{\prime \prime} 4\right)$. This exceeds the temperature of $\mathrm{H}$ II regions $\left(\sim 10^{4} \mathrm{~K}\right)$ and hence suggests the dominance of non-thermal synchrotron emission. The second constraint is from the radio flux densities. The nucleus has $1.49 \mathrm{GHz}(20 \mathrm{~cm})$ and $4.86 \mathrm{GHz}(6 \mathrm{~cm})$ flux densities of $38.5 \mathrm{mJy}$ and $26.1 \mathrm{mJy}$, respectively, from a source with a $\lesssim 0^{\prime \prime} 4$ size (Condon et al. 1990; Baan \& Klöckner 2006); the resolutions of these observations are about $0{ }^{\prime \prime} 4$ and $00^{\prime} 15$, respectively. The $20 \mathrm{~cm}$ luminosity of the nucleus is $5.3 \times 10^{21} \mathrm{~W} \mathrm{~Hz}^{-1}$ assuming isotropy of the radio emission. The third constraint is the spectral index between 20 and $6 \mathrm{~cm}, \alpha_{20-6 \mathrm{~cm}}=-0.33$ (for $\left.S \propto v^{\alpha}\right)$. It is closer to the spectral index of thermal free-free emission $(\alpha=-0.1)$ than to that of non-thermal emission $(\alpha \approx-0.8)$ both from optically thin star-forming regions (e.g., Turner \& Ho 1994; Murphy et al. 2011). The apparent inconsistency between the first and third constraints can be reconciled if the radio emission is partly opaque, i.e., if either synchrotron self-absorption or free-free absorption by thermal plasma is significant. We therefore re-derived the $20 \mathrm{~cm}$ flux density using the $6 \mathrm{~cm}$ data and a non-thermal spectral index of -0.8 , while noting that this simplified correction will be insufficient if the $6 \mathrm{~cm}$ emission is also opaque. The corrected $20 \mathrm{~cm}$ flux density and luminosity are $67.2 \mathrm{mJy}$ and $9.3 \times 10^{21} \mathrm{~W} \mathrm{~Hz}^{-1}$, respectively. The SFR of $\gtrsim 9 M_{\odot} \mathrm{yr}^{-1}$ is from this $20 \mathrm{~cm}$ luminosity and formula (14) of Murphy et al. (2011), which in our case is $\left(\mathrm{SFR} / M_{\odot} \mathrm{yr}^{-1}\right)=6.64 \times 10^{-22}(v / \mathrm{GHz})^{-0.8}\left(L_{v} / \mathrm{W} \mathrm{Hz}^{-1}\right)$. This formula was derived using the proportionality of supernova rate to the SFR (adopting the ratio between them from Starburst99) and the empirical relation between the supernova rate and the non-thermal radio luminosity. A similar SFR was derived in $\mathrm{C} 12$ from radio data albeit without the opacity correction.

The radio-derived SFR is smaller than needed for the starburst model at the face value. This may be for the following (at least) two reasons if the starburst model is correct. First, the radio SFR is a lower limit and can be higher as mentioned above. Second, the SFR may be underestimated because the starburst is very young and is still deficient of supernovae compared to older starbursts for which the SFR formula above was derived. This nascent starburst is what Roussel et al. (2003) argued for from the relative weakness of radio emission compared to the infrared emission in this galaxy and by analyzing radio data in a way similar to ours except that they used lower-resolution data (beam $\left.\geqslant 45^{\prime \prime}\right)$. An obvious weakness of this second explanation is that the dominance of non-thermal emission (our first constraint) does not accord with the very young starburst. The radio emission should be dominated by thermal emission from H II regions rather than by non-thermal emission from supernovaeinduced cosmic rays as long as the nucleus is optically thin at radio wavelengths. One could still attribute the weak thermal emission to direct absorption of ionizing photons by dust and to high-opacity of the free-free emission. Non-thermal synchrotron emission can be less affected by these owing to the higher penetrating power of cosmic rays than ionizing photons. These qualitative explanations are not particularly attractive because of their complexity but, we do not yet have a proof to rule them out.

In summary, the lower-limit SFR derived from radio data under the starburst-dominant model is about a factor of three smaller than the lower-limit SFR needed for the luminosity of the nucleus. Although various uncertainties outlined above prevent us from excluding the starburst model, an energetically 
dominant starburst would need an elaborate model to explain the radio observations.

\subsubsection{Implications for Previous Studies}

We also note implications of our new observations to a previous study of this energy source problem. Full reanalysis of all existing observations in light of our new data is beyond the scope of this paper.

Imanishi et al. (2004) used the presumed lack of shocks in the nucleus of this non-merging galaxy and the high HCN-to$\mathrm{HCO}^{+}$intensity ratio that they obtained toward the nucleus to infer that the warm $\mathrm{H}_{2}$ they observed in the nucleus is due to $\mathrm{X}$-ray heating. They then estimated the AGN luminosity from the $\mathrm{H}_{2}$ line luminosity and reached a conclusion that the AGN is the dominant energy source in the nucleus. Our discovery of the red cone that we attribute to an outflow from the nucleus, as well as the discovery by González-Alfonso et al. (2012b) of the blueshifted absorption presumably due to an inflow, raise the possibility of shock contribution to the infrared $\mathrm{H}_{2}$ lines. The $\mathrm{HCN}$-to- $\mathrm{HCO}^{+}$ratio does not give us the fraction of $\mathrm{H}_{2}$ luminosity due to an $\mathrm{AGN}$. Regarding the $\mathrm{HCN}-$ to- $\mathrm{HCO}^{+}$ratio itself, while its enhancement around AGN gained support from more recent observations at kpc resolutions (Krips et al. 2008), it has been also found to vary within the center of a single galaxy from one giant molecular cloud to another in the range covered by the low-resolution observations of starbursts and AGNs (Meier \& Turner 2012). The latter authors suggested shock enhancement of $\mathrm{HCN}$ citing stronger enhancement of $\mathrm{HCN}$ than $\mathrm{HCO}^{+}$in the molecular outflow from a protostar (Bachiller \& Perez Gutierrez 1997). In addition, for the particular case of NGC 4418, our detection and analysis of rotational lines from vibrationally excited HCN toward the nucleus (S10) further complicate the line ratio analysis by introducing the excitation path through the vibrationally excited state. At present, no estimate exists for the fractional contributions of AGN $\mathrm{X}$-rays and other mechanisms to the observed high $\mathrm{HCN} / \mathrm{HCO}^{+}$ ratio. Thus, these recent observations make the assumed $100 \%$ contribution of X-ray heating to the $\mathrm{H}_{2}$ line luminosity uncertain. Therefore, the AGN luminosity estimated from the $\mathrm{H}_{2}$ line luminosity is more uncertain than it was at the time Imanishi et al. (2004) interpreted their data. This is not to say that the new observations are against the AGN hypothesis because the AGN dominance can probably still explain the observations and there are new observations that prefer an AGN for molecular chemistry in the nucleus (González-Alfonso et al. 2012a). As was the case in the radio analysis reviewed above, we have a problem that contributions of multiple (possible) mechanisms to observed properties make a conclusive data interpretation difficult. It seems that we still need to accumulate more constraints to obtain the breakdown of the luminosity sources in the nucleus.

\subsection{The Gas/Dust Shroud of the Nucleus and Comparison with Other Observations}

The shroud of the $10^{11} L_{\odot}$ source is a $\sim 20$ pc core having $\tau_{860 \mu \mathrm{m}} \sim 1$ and the surrounding $\gtrsim 100 \mathrm{pc}$ gas concentration that is generally warmer and denser toward the center. The Comptonthick core has a surface temperature $\sim 150 \mathrm{~K}$ and a mean density $\sim 5 \times 10^{5} \mathrm{H}_{2} \mathrm{~cm}^{-3}$ (Section 3.3.2). The radial variation of gas properties is indicated by the emission size sequence of "CO $>\mathrm{HCN}, \mathrm{HCO}^{+}>$dust continuum" (Section 3.2), the continuum properties suggestive of "dense core + diffuse envelope" structure (Sections 3.3.1 and 3.3.4), the larger velocity widths of lines with higher critical densities (Section 3.4), and the dependence of line ratios and equivalent widths on area size (Section 3.5). The same emission size sequence was observed in Arp 220 (Sakamoto et al. 2009). The declining temperature gradient from the center to larger radii has been an explanation for the deep $9.7 \mu \mathrm{m}$ depression in the mid-IR spectrum (Section 1.1).

Our observations on the properties of the central gas concentration are generally consistent with those from the $1 \mathrm{~mm}$ observations in our companion paper $(\mathrm{C} 12)$. Leaving the comparison between the $1 \mathrm{~mm}$ and submillimeter observations to that paper, we only mention that the deconvolved $\mathrm{CO}(2-1)$ peak brightness temperature in $\mathrm{C} 12$ is $80 \mathrm{~K}$ to agree with the $\mathrm{CO}(3-2)$ peak $T_{\mathrm{b}}$ of $90 \mathrm{~K}$ and that $\mathrm{C} 12$ also reports radial variation of gas properties.

González-Alfonso et al. (2012b) made a detailed analysis of the circumnuclear gas properties using Herschel spectroscopy at 50-200 $\mu \mathrm{m}$. They detected dozens of high-excitation lines, mostly in absorption, from $\mathrm{H}_{2} \mathrm{O}, \mathrm{OH}, \mathrm{O}$, $\mathrm{HCN}, \mathrm{NH}_{3}$, and their ${ }^{18} \mathrm{O}$ isotopologues at $100-300 \mathrm{~km} \mathrm{~s}^{-1}$ resolutions. Using these lines, they made a multi-component model in which the ISM is warmer in more compact components. Our observations are consistent with this trend. There are also agreements on the dust temperature around $150 \mathrm{~K}$ at $r \sim 10 \mathrm{pc}$ and on the higher gas densities in more compact components down to the $r \sim 10 \mathrm{pc}$ component (according to $\left(\tau_{\text {dust }, 200 \mu \mathrm{m}} / \mathrm{size}\right)$ in their Table 1$)$. A difference between their model and our observations is that we detected about $0.2 \mathrm{Jy}$ of $860 \mu \mathrm{m}$ continuum from a $\sim 0^{\prime \prime} 1$ $(20 \mathrm{pc})$ core, whereas their model predicts a quarter of that from a component of the same size and about the same temperature $(150 \mathrm{~K})$. Our larger flux density and brightness temperature lead us to a larger dust opacity for the core at $860 \mu \mathrm{m}$. In order for the 50-200 $\mu \mathrm{m}$ and $860 \mu \mathrm{m}$ observations to be consistent, it may be that the ISM in and around the core is not uniform to allow more leakage of emission from the warmer inner region than expected in the case of uniform shells. Another possibility is that the dust $\beta$ is smaller than 2. González-Alfonso et al. (2012b) also obtained an oxygen isotope abundance comparable to that in the solar neighborhood, $\left[{ }^{16} \mathrm{O} /{ }^{18} \mathrm{O}\right] \sim 500$. As they noted, the lack of ${ }^{18} \mathrm{O}$ enhancement through stellar processing is in accord with the luminosity source being an AGN and/or young pre-supernova starburst.

\subsection{Evolution of the Luminous Nucleus}

The formation and evolution of the hidden luminous nucleus must be closely linked to those of the massive gas concentration at the nucleus. It is because the concentrated gas affects the luminosity source and its appearance through fueling and shielding.

\subsubsection{What Drove the Large Amount of Gas to the Nucleus?}

The optical morphology of NGC 4418 (Figure 1) does not show significant disturbance or distortion to suggest a major merger. There is a blue companion VV655 at 3' southeast ( $30 \mathrm{kpc}$ on the sky) and at about the same redshift $\left(2200 \mathrm{~km} \mathrm{~s}^{-1}\right){ }^{9}$ Although the two are probably physically associated with each other, the large separation is unlike that in Arp 220 whose merger nuclei have a projected separation of only $0.3 \mathrm{kpc}$. For Arp 220 there is no doubt that the concentration of a large amount of gas to each nucleus was a consequence of the dynamical

\footnotetext{
9 What is cataloged in the atlas of interacting galaxies by Vorontsov-Velyaminov (1977) is this companion itself and not the pair of
} VV655 and NGC 4418. 
perturbation of the major merger. In contrast, NGC 4418 does not appear to have acquired its nuclear gas concentration, and hence the nuclear luminosity, through a major merger although some properties of the nucleus are similar to those in Arp 220.

A stellar bar and minor merger are milder mechanisms for gas concentration than major merger and galaxy interaction. For example, stellar bars are known to transport cold gas in galaxy disks, sometimes as much as $>10^{8} M_{\odot}$, to the galactic centers (Sakamoto et al. 1999). If the gas concentration in NGC 4418 were due to a stellar bar the situation may be similar to the one in the barred spiral galaxy NGC 3504 that has a single-peaked concentration of molecular gas with a scale length of $220 \mathrm{pc}$ and a mass of $10^{8} M_{\odot}$ in the central 200 pc (Kenney et al. 1993). The morphological classification of NGC 3504, (R)SAB(s)ab, is very close to that of NGC 4418 (Table 1) including the same moderate bar-type distortion. Currently, our data of NGC 4418 show neither a kinematical sign of bar-driven gas dynamics nor any clear sign of recent minor merger, although such signs may be found in the future in the somewhat disorderly gas motion in the central kpc (Figure 7).

The nuclear gas concentration may also be because most of the gas came from bulge stars. The gas originated from the galactic bulge through stellar winds and supernovae explosions would have little net angular momentum. Therefore, some of it will sink to the galactic center when cooled. A stellar population with a normal IMF returns about $30 \%$ of its mass to the interstellar space in $10^{10} \mathrm{yr}$ (Jungwiert et al. 2001). The current bulge mass of NGC 4418 is about $0.6 \times 10^{10} M_{\odot}$, where we used the $K$-band bulge luminosity in Section 3.4 and the same $L / M$ as in Section 4.1.3. The cumulative mass loss from the bulge stars should therefore be about $3 \times 10^{9} M_{\odot}$. Stars formed from this gas should have small specific angular momentum and hence further mass loss from them should keep the low specific angular momentum. The $\sim 10^{9} M_{\odot}$ molecular gas currently concentrated to the nucleus may be a fraction of the low angular momentum gas originated this way from the bulge stars. (See Young et al. 2011; Davis et al. 2011 and references therein for the general molecular gas content in local early-type galaxies and discussion on their origins.)

We note two more galaxies that have been recently found to host a compact and massive concentration of molecular gas at their centers. NGC 1377 is an isolated lenticular galaxy that shares infrared characteristics such as the deep $9.7 \mu \mathrm{m}$ absorption with NGC 4418. Aalto et al. (2012) found a singlepeaked $\sim 100 \mathrm{pc}$ scale concentration of molecular gas at the nucleus and estimated a total gas mass in the central $400 \mathrm{pc}$ of $\sim 10^{8} M_{\odot}$. NGC 1266 is another lenticular galaxy that has a strong concentration of molecular gas $\left(\sim 10^{8.5} M_{\odot}\right)$ in the central $100 \mathrm{pc}$ without a clear sign of external disturbance or bar-driven gas transport (Alatalo et al. 2011). There may be a common mechanism to cause the compact massive gas concentrations in these galaxies and NGC 4418. It may be the bulge origin of the gas as mentioned above since these galaxies have large bulge fractions.

\subsubsection{Ongoing Evolution}

Our discovery of an outflow feature from the center of NGC 4418 makes the nucleus to have both an outflow and a previously known inflow. The inflow has been seen on our sightline near the galactic plane (Section 3.4) and the outflow is perpendicular to the galactic plane (at least at $>500$ pc scales). Such complex gas motion has been modeled around a galaxy nucleus hosting both starburst and an active nucleus (e.g., Wada \& Norman 2002; Wada 2012). Ohsuga et al. (2005) also simulated an outflow + inflow system at a smaller scale around a black hole and showed that the system can achieve the Eddington luminosity or higher. While we tentatively estimate the mass outflow rate to be $\sim 4 M_{\odot} \mathrm{yr}^{-1}$, González-Alfonso et al. (2012b) estimated an inflow rate of $\lesssim 12 M_{\odot} \mathrm{yr}^{-1}$ from the redshifted absorption lines they found. The latter must be an overestimate because it assumed a spherically symmetric inflow without knowing the outflow. It is therefore possible that the net outflow rate is close to zero or even positive.

The outflow from the deeply embedded nucleus may be driven at least at its root by the radiation pressure on dust from the luminous nucleus (Scoville 2003; Murray et al. 2005). The criterion for a radiation-driven outflow is given with the $L / M$ as

$$
\frac{L_{\mathrm{bol}}}{M_{\mathrm{dyn}}}>\frac{4 \pi c G}{\kappa},
$$

where $\kappa$ is the mass opacity coefficient, $c$ is the speed of light, and $G$ is the gravitational constant. For fully ionized hydrogen plasma, $\kappa$ is the ratio of the Thomson cross section to the masses of proton and electron and is $\sigma_{\mathrm{T}} /\left(m_{\mathrm{p}}+m_{\mathrm{e}}\right)=0.4 \mathrm{~cm}^{2} \mathrm{~g}^{-1}$. The minimum $L / M$ for the outflow is thus the Eddington limit, $3.3 \times 10^{4} L_{\odot} M_{\odot}{ }^{-1}$. For $3 \mu \mathrm{m}$ mass opacity coefficient of $50 \mathrm{~cm}^{2} \mathrm{~g}^{-1}$ (Scoville 2003), where the opacity is due to dust, the minimum $L / M$ for the outflow is $300 L_{\odot} M_{\odot}{ }^{-1}$. For $50 \mu \mathrm{m}$ where the SED of NGC 4418 peaks, the mass opacity coefficient is $\kappa=2.5 \mathrm{~cm}^{2} \mathrm{~g}^{-1}$ (Hildebrand 1983) and the minimum $L / M$ for the outflow is $5000 L_{\odot} M_{\odot}{ }^{-1}$. From these, a radiationdriven outflow appears possible from the region where the peak of the SED is in near-IR; the peak shifts to longer wavelength further from the central source. Unlike the inner region where the radiation pressure may drive the outflow, the outer region of the outflow along the rotation axis of the galaxy may be driven by thermal pressure since the polar direction is the direction of the steepest pressure gradient.

With the inflow/outflow rates of several $M_{\odot} \mathrm{yr}^{-1}$ the ongoing evolution of the circumnuclear gas concentration is rapid in the sense that most of the $\sim 10^{8} M_{\odot}$ molecular gas in the central $20 \mathrm{pc}$ will be replaced in $10 \mathrm{Myr}$. Although the presumed accreting disk + outflow structure may stay longer with a continuous supply of gas from the larger gas reservoir outside the $20 \mathrm{pc}$ core, the degree of nuclear obscuration may significantly vary in the gas-flow timescale. The currently high $L / M$ can also change by an order of magnitude in $10 \mathrm{My}$ for the starburst case and by a larger factor in much shorter time in the AGN case. These considerations suggest a rapid evolution and a short duration of the current state of the NGC 4418 nucleus.

Finally, NGC 4418 and the two galaxies mentioned in Section 4.3.1 for their nuclear gas concentrations share an outflow of molecular gas and/or dust from each nucleus. The nuclear outflow from NGC 1377 is seen in CO kinematics (Aalto et al. 2012) and, as in NGC 4418, as a red cone on a semiminor axis in an optical color-index image (Heisler \& Vader 1994). Alatalo et al. (2011) found the outflow of NGC 1266 from observations of $\mathrm{CO}, \mathrm{H} \mathrm{I}$, and ionized gas and suggested it to be AGN-driven. There may be a common evolutionary relation between the compact nuclear gas concentrations and the outflows in these galaxies because a gas concentration is generally expected to feed nuclear activities that can drive an outflow. 


\section{SUMMARY AND CONCLUSIONS}

We made high-resolution 860 and $450 \mu \mathrm{m}$ observations of NGC 4418, a luminous infrared galaxy having a deeply embedded nucleus. Continuum and lines including $\mathrm{CO}(3-2)$, $\mathrm{HCN}(4-3), \mathrm{HCO}^{+}(4-3)$, and $\mathrm{CS}(7-6)$ in the $350 \mathrm{GHz}$ band were imaged at resolutions from $0^{\prime \prime} 3$ to $1^{\prime \prime}(=165 \mathrm{pc})$ in the central $34^{\prime \prime}(5.6 \mathrm{kpc})$. Continuum at $660 \mathrm{GHz}(450 \mu \mathrm{m})$ was imaged at 0.2 resolution. We also examined SDSS optical images for circumnuclear features related to the luminous nucleus. Our key results are the following.

1. The ISM traced by the submillimeter emission is found strongly concentrated toward the nucleus. $\mathrm{CO}(3-2)$ emission is detected only in the central $1 \mathrm{kpc}\left(6^{\prime \prime}\right)$ of the galaxy with almost all the single-dish flux. More than half of the CO flux in the central kpc is in the central $165 \mathrm{pc}\left(1^{\prime \prime}\right)$. Higher excitation lines other than $\mathrm{CO}$ are more concentrated toward the nucleus (FWHM $\sim 50 \mathrm{pc}=0^{\prime}$ ' 3 ), and the $860 \mu \mathrm{m}$ continuum emission is even more compact.

2. The peak brightness temperature of $\mathrm{CO}(3-2)$ in the nuclear gas concentration is as high as $90 \mathrm{~K}$ at $0^{\prime} .3(50 \mathrm{pc})$ resolution, indicating the presence of hot molecular gas in the nucleus. Other indications of hot or highly excited molecular gas are lines from vibrationally excited $\mathrm{HCN}$ and $\mathrm{HC}_{3} \mathrm{~N}$ with upper energy levels at $660-1070 \mathrm{~K}$ above the ground.

3. The $860 \mu \mathrm{m}$ continuum emission at the nucleus has a size of $\sim 00^{\prime} 1$ (17 pc) in FWHM and deconvolved peak brightness temperature $\left(T_{\mathrm{b}}\right)$ of about $210 \mathrm{~K}$ for a Gaussian model. The diameter is $27 \mathrm{pc}$ and $T_{\mathrm{b}} \sim 120 \mathrm{~K}$ for a uniform disk (sphere) model. The $860 \mu \mathrm{m}$ opacity of this core is on the order of one and therefore the core is Compton thick $\left(N_{\mathrm{H}} \gtrsim 10^{25} \mathrm{~cm}^{-2}\right)$. The bolometric luminosity of this dusty core is estimated from these parameters to be $L_{\text {bol }} \sim 10^{11.0} L_{\odot}$. Most of the galaxy luminosity $\left(10^{11.1} L_{\odot}\right)$ is from this core. Its luminosity surface density is $\Sigma\left(L_{\mathrm{bol}}\right) \sim 10^{8.5 \pm 0.5} L_{\odot} \mathrm{pc}^{-2}$.

4. Continuum emission at $450 \mu \mathrm{m}$ also peaks at the nucleus but its flux density in our $30 \mathrm{pc}(\approx 0$ '2) resolution data is less than half of the total flux density from single-dish observations. A more extended region than the $\sim 0^{\prime} .1$ core, presumably the region detected in $\mathrm{CO}(3-2)$, must have significant $450 \mu \mathrm{m}$ emission. Extinction of the core by this colder envelope is a likely additional reason for the small core contribution at $450 \mu \mathrm{m}$.

5. The dynamical mass within the central $30 \mathrm{pc}$ is estimated to be $\sim 2 \times 10^{8} M_{\odot}$ from the molecular gas velocities in the central 0.5. The nucleus thus has $L_{\mathrm{bol}} / M_{\mathrm{dyn}} \sim$ $500 L_{\odot} / M_{\odot}$. Also, higher excitation lines are found broader than lower excited ones in the nucleus. This is likely due to faster gas motion and higher gas excitation parameters (e.g., density and temperature) toward the center. The CO line at $r=00^{\prime} 25-3^{\prime \prime}$ (40-500 pc) shows complex velocity structure that likely contains radial motion in addition to rotation.

6. The observed high $L / M, \Sigma(L)$, and $N_{\mathrm{H}}$ as well as the nuclear mass budget are consistent with a hidden AGN to be the main luminosity source while they also allow a young starburst. This confirms the proposition of Roche et al. (1986) and subsequent researchers. A dominant AGN would have a high Eddington ratio $\sim 0.3$ for the black hole expected from the bulge luminosity. For star formation to achieve the observed $L / M$ and $\Sigma\left(L_{\mathrm{bol}}\right)$, there must be a compact concentration of $\lesssim 5 \mathrm{Myr}$ old stars well within the central $20 \mathrm{pc}$ with a total mass on the order of $10^{8} M_{\odot}$. Submillimeter data of higher quality will further constrain the nature of the nucleus.

7. We found in the optical data a U-shaped red feature along a semi-minor axis of the galaxy. We suggest it to be an outflow-induced cone seen in dust extinction (i.e., reddening). We estimate the mass of the outflowing gas to be $\sim 4 \times 10^{7} M_{\odot}$ and the rate of mass outflow from the nucleus to be $\sim 4 M_{\odot} \mathrm{yr}^{-1}$, although the latter is tentative. The luminous nucleus is blowing out its shroud in the polar direction while it is still fed from another direction as suggested from the previously known redshifted absorption. Because these radial flows can replace most gas in the $\sim 20 \mathrm{pc}$ core in only $10^{7} \mathrm{yr}$, they should play a significant role in the evolution of the luminous nucleus.

We thank the SMA staff for making these observations possible and Mark Gurwell for helping flux calibration. K.S. thanks Dr. Eduardo González-Alfonso for answering questions about his recent work on NGC 4418. This research made use of the NASA/IPAC Extragalactic Database (NED), NASA's Astrophysics Data System (ADS), the splatalogue database for astronomical spectroscopy, the Sloan Digital Sky Survey (SDSS), and the JPL HORIZONS system. K.S. was supported by the Taiwanese National Science Council grant 99-2112-M001-011-MY3. S.M. acknowledges the co-funding of this work under the Marie Curie Actions of the European Commission (FP7-COFUND).

Facilities: SMA, Sloan

\section{REFERENCES}

Aalto, S., Monje, R., \& Martín, S. 2007, A\&A, 475, 479

Aalto, S., Muller, S., Sakamoto, K., et al. 2012, A\&A, 546, A68

Alatalo, K., Blitz, L., Young, L. M., et al. 2011, ApJ, 735, 88

Albrecht, M., Krügel, E., \& Chini, R. 2007, A\&A, 462, 575

Andrews, B. H., \& Thompson, T. A. 2011, ApJ, 727, 97

Armus, L., Heckman, T., \& Miley, G. 1987, AJ, 94, 831

Baan, W. A., \& Klöckner, H.-R. 2006, A\&A, 449, 559

Bachiller, R., \& Perez Gutierrez, M. 1997, ApJL, 487, 93

Brauher, J. R., Dale, D. A., \& Helou, G. 2008, ApJS, 178, 280

Bridle, A. H., \& Greisen, E. W. 1994, NRAO AIPS Memo, 87

Chung, A., Yun, M. S., Naraynan, G., Heyer, M., \& Erickson, N. R. 2011, ApJL, 732,15

Condon, J. J., Helou, G., Sanders, D. B., \& Soifer, B. T. 1990, ApJS, 73, 359

Costagliola, F., \& Aalto, S. 2010, A\&A, 515, A71

Costagliola, F., Aalto, S., Sakamoto, K., et al. 2012, A\&A, submitted (C12)

Dale, D. A., Sheth, K., Helou, G., Regan, M. W., \& Hüttemeister, S. 2005, AJ, 129,2197

Davis, T. A., Alatalo, K., Sarzi, M., et al. 2011, MNRAS, 417, 882

de Grijp, M. H. K., Miley, G. K., Lub, J., \& de Jong, T. 1985, Natur, 314, 240

de Vaucouleurs, G., de Vaucouleurs, A., Corwin, H. G., et al. 1991, Third

Reference Catalogue of Bright Galaxies (Berlin: Springer)

Dudley, C. C., \& Wynn-Williams, C. G. 1997, ApJ, 488, 720

Duncan, W. D., Robson, E. I., Ade, P. A. R., Sandell, G., \& Griffin, M. J. 1990, MNRAS, 243, 126

Dunne, L., \& Eales, S. A. 2001, MNRAS, 327, 697

Dunne, L., Eales, S., Edmunds, M., et al. 2000, MNRAS, 315, 115

Evans, A. S., Becklin, E. E., Scoville, N. Z., et al. 2003, AJ, 125, 2341

Fischer, J., Sturm, E., González-Alfonso, E., et al. 2010, A\&A, 518, L41

González-Alfonso, E., Fischer, J., Bruderer, S., et al. 2012a, arXiv:1211.5064

González-Alfonso, E., Fischer, J., Graciá-Carpio, J., et al. 2012b, A\&A, 541, A4

Graciá-Carpio, J., Sturm, E., Hailey-Dunsheath, S., et al. 2011, ApJL, 728, 7

Graham, A. W., \& Worley, C. C. 2008, MNRAS, 388, 1708

Heisler, C. A., \& Vader, J. P. 1994, AJ, 107, 35

Hildebrand, R. H. 1983, QJRAS, 24, 267

Ho, L. C. 2008, ARA\&A, 46, 475

Ho, P. T. P., Moran, J. M., \& Lo, K. Y. 2004, ApJL, 616, 1

Hopkins, P. F., Hernquist, L., Cox, T. J., et al. 2006, ApJS, 163, 1 
Hunter, S. D., Bertsch, D. L., Catelli, J. R., et al. 1997, ApJ, 481, 205 Imanishi, M., Imase, K., Oi, N., \& Ichikawa, K. 2011, AJ, 141, 156 Imanishi, M., Nakanishi, K., Kuno, N., \& Kohno, K. 2004, AJ, 128, 2037

Jarrett, T. H., Chester, T., Cutri, R., et al. 2000, AJ, 119, 2498

Jungwiert, B., Combes, F., \& Palouš, J. 2001, A\&A, 376, 85

Kawara, K., Taniguchi, Y., Nakai, N., \& Sofue, Y. 1990, ApJL, 365, 1

Keene, J., Hildebrand, R. H., \& Whitcomb, S. E. 1982, ApJL, 252, 11

Kenney, J. D. P., Carlstrom, J. E., \& Young, J. S. 1993, ApJ, 418, 687

Kennicutt, R. C., Jr. 1998, ARA\&A, 36, 189

Kennicutt, R. C., \& Evans, N. J. 2012, ARA\&A, 50, 531

Kewley, L. J., Heisler, C. A., Dopita, M. A., et al. 2000, ApJ, 530, 704

Krips, M., Neri, R., García-Burillo, S., et al. 2008, ApJ, 677, 262

Kroupa, P. 2002, Sci, 295, 82

Kwan, J., \& Scoville, N. 1976, ApJ, 209, 102

Lahuis, F., Spoon, H. W. W., Tielens, A. G. G. M., et al. 2007, ApJ, 659, 296

Lehnert, M. D., \& Heckman, T. M. 1995, ApJS, 97, 89

Leitherer, C., Ortiz Otálvaro, P. A., Bresolin, F., et al. 2010, ApJS, 189, 309

Leitherer, C., Schaerer, D., Goldader, J. D., et al. 1999, ApJS, 123, 3

Lisenfeld, U., Isaak, K. G., \& Hills, R. 2000, MNRAS, 312, 433

Maiolino, R., Comastri, A., Gilli, R., et al. 2003, MNRAS, 344, L59

Malhotra, S., Helou, G., Stacey, G., et al. 1997, ApJL, 491, 27

Marconi, A., \& Hunt, L. K. 2003, ApJL, 589, 21

Matsushita, S., Iono, D., Petitpas, G. R., et al. 2009, ApJ, 693, 56

Meier, D. S., \& Turner, J. L. 2012, ApJ, 755, 104

Mould, J. R., Huchra, J. P., Freedman, W. L., et al. 2000, ApJ, 529, 786

Murphy, E. J., Condon, J. J., Schinnerer, E., et al. 2011, ApJ, 737, 67

Murray, N. 2009, ApJ, 691, 946

Murray, N., Quataert, E., \& Thompson, T. A. 2005, ApJ, 618, 569

Ohsuga, K., Mori, M., Nakamoto, T., \& Mineshige, S. 2005, ApJ, 628, 368

Roche, P. F., Aitken, D. K., Smith, C. H., \& James, S. D. 1986, MNRAS, 218, 19P

Roche, P. F., \& Chandler, C. J. 1993, MNRAS, 265, 486

Roussel, H., Helou, G., Beck, R., et al. 2003, ApJ, 593, 733

Sakamoto, K. 2012, in ASP Conf. Ser., Galaxy Mergers in an Evolving Universe, in press (arXiv:1207.3678)

Sakamoto, K., Aalto, S., Evans, A. S., Wiedner, M. C., \& Wilner, D. J. 2010, ApJL, 725, 228 (S10)

Sakamoto, K., Aalto, S., Wilner, D. J., et al. 2009, ApJL, 700, 104

Sakamoto, K., Mao, R.-Q., Matsushita, S., et al. 2011, ApJ, 735, 19

Sakamoto, K., Okumura, S. K., Ishizuki, S., \& Scoville, N. Z. 1999, ApJ, 525,691
Sakamoto, K., Wang, J., Wiedner, M. C., et al. 2008, ApJ, 684, 957 (S08)

Sanders, D. B., Mazzarella, J. M., Kim, D.-C., Surace, J. A., \& Soifer, B. T. 2003, AJ, 126, 1607

Sanders, D. B., \& Mirabel, I. F. 1996, ARA\&A, 34, 749

Sanders, D. B., Scoville, N. Z., \& Soifer, B. T. 1991, ApJ, 370, 158

Sanders, D. B., Soifer, B. T., Elias, J. H., Neugebauer, G., \& Matthews, K. 1988, ApJL, 328, 35

Sault, R. J., Teuben, P. J., \& Wright, M. C. H. 1995, in ASP Conf. Ser. 77 Astronomical Data Analysis Software and Systems IV, ed. R. Shaw et al. (San Francisco, CA: ASP), 433

Schöier, F. L., van der Tak, F. F. S., van Dishoeck, E. F., \& Black, J. H. 2005, A\&A, 432, 369

Scoville, N. 2003, JKAS, 36, 167

Scoville, N. Z., Carlstrom, J. E., Chandler, C. J., et al. 1993, PASP, 105, 1482

Seaquist, E., Yao, L., Dunne, L., \& Cameron, H. 2004, MNRAS, 349, 1428

Siebenmorgen, R., Haas, M., Pantin, E., et al. 2008, A\&A, 488, 83

Smith, H. E., Lonsdale, C. J., \& Lonsdale, C. J. 1998, ApJ, 492, 137

Soifer, B. T., Bock, J. J., Marsh, K., et al. 2003, AJ, 126, 143

Soifer, B. T., Neugebauer, G., Matthews, K., et al. 2000, AJ, 119, 509

Soifer, B. T., Neugebauer, G., Matthews, K., et al. 2001, AJ, 122, 1213

Soifer, B. T., Neugebauer, G., Matthews, K., Egami, E., \& Armus, L. 2004, PASP, 116, 493

Spoon, H. W. W., Keane, J. V., Tielens, A. G. G. M., Lutz, D., \& Moorwood, A. F. M. 2001, A\&A, 365, L353

Spoon, H. W. W., Marshall, J. A., Houck, J. R., et al. 2007, ApJL, 654, 49

Terzić, B., \& Graham, A. W. 2005, MNRAS, 362, 197

Thompson, T. A., Quataert, E., \& Murray, N. 2005, ApJ, 630, 167

Turner, J. L., \& Ho, P. T. P. 1994, ApJ, 421, 122

Vázquez, G. A., \& Leitherer, C. 2005, ApJ, 621, 695

Vorontsov-Velyaminov, B. A. 1977, A\&AS, 28, 1

Wada, K. 2012, ApJ, 758, 66

Wada, K., \& Norman, C. A. 2002, ApJL, 566, 21

Weiner, B. J., Coil, A. L., Prochaska, J. X., et al. 2009, ApJ, 692, 187

Worthey, G. 1994, ApJS, 95, 107

Wynn-Williams, C. G., \& Becklin, E. E. 1993, ApJ, 412, 535

Yang, M., \& Phillips, T. 2007, ApJ, 662, 284

Yao, L., Seaquist, E. R., Kuno, N., \& Dunne, L. 2003, ApJ, 588, 771

Young, J. S., Xie, S., Kenney, J. D. P., \& Rice, W. L. 1989, ApJS, 70, 699

Young, J. S., Xie, S., Tacconi, L., et al. 1995, ApJS, 98, 219

Young, L. M., Bureau, M., Davis, T. A., et al. 2011, MNRAS, 414, 940

Yun, M. S., Reddy, N. A., \& Condon, J. J. 2001, ApJ, 554, 803 